\title{
Coherence approach to neutron polarization propagation in instruments
}

\author{
Victor O. de Haan,* Ad A. van Well, and Jeroen Plomp \\ Department Radiation, Radionuclides and Reactors, Faculty of Applied Sciences, Delft University of Technology, \\ Mekelweg 15, 2629 JB Delft, The Netherlands \\ (Received 6 December 2007; published 27 March 2008)
}

\begin{abstract}
The propagation of the mutual coherence function is a well known method to describe the effects of neutron's propagation through scattering instruments. This method is extended with the description of the coherence matrix to account for neutron polarization effects and its propagation through an instrument. A propagation law for the coherence matrix and the condition under which it can be used is derived. A rigorous description of polarization analysis is given. The phase-object approximation to describe small angle neutron scattering is extended to incorporate scattering in magnetic materials. The developed theory is applied to spin echo small angle neutron scattering and the neutron depolarization technique.
\end{abstract}

DOI: 10.1103/PhysRevB.77.104121

PACS number(s): 42.25.Fx, 42.25.Gy, 42.25.Hz, 42.25.Ja

\section{INTRODUCTION}

When general properties are assigned to functions governed by a wave equation, coherence theory can be applied. Then, it is possible to find general formulations for the propagation of wave functions despite that their physical background might be different. Coherence theory is widely used in optical ${ }^{1}$ scattering phenomena but is still fairly new in $\mathrm{x}-$ ray $^{2-4}$ or neutron scattering. Textbooks briefly discuss the coherence properties of neutron beams ${ }^{5}$ but do not apply it to neutron scattering. Rauch et al. ${ }^{6}$ used the results of coherence theory to explain their neutron-interferometry measurements.

Recently Gähler et al. used a space-time approach to derive the neutron scattering formulas for many body systems ${ }^{7}$ in the kinematic approximation. In the following, this approach is followed and extended to the phase-object approximation $^{8}$ for small angle neutron scattering (SANS). Further, coherence theory is used to describe neutron polarization effects ${ }^{9}$ and the way a polarized beam is transported through an instrument. This enables a coherent approach to these effects without the need for some ad hoc definition on beam polarization and analysis.

Coherence theory can be interpreted as a shorthand notation for the description of ensemble averages of wave functions and their (mathematical) properties. In general, coherence theory does not give new insight into the involved physics. Worse, it complicates the classical view of a neutron as a small magnet moving through the instrument performing Larmor precession in magnetic flux density regions. However, it gives possibilities to apply insight from one field of physics to another. The main advantage of coherence theory is that it gives the possibility to calculate the results of the propagation of the wave function through the instrument directly. No ad hoc folding of theoretical results with instrumental resolution is needed, but direct computation of measurable data is possible. Coherence theory seems to be quite complicated and unnecessary to describe the neutron propagation through an instrument. However, after getting used to the concept of the mutual coherence function and the way this function is propagated through free space and space with magnetic flux density, it gives a more thorough account of the important effects before and after the scattering process.

This paper is organized as follows. Section II describes the basic principles of coherence theory as applied to neutron wave fields having interaction with magnetic fluxes. It introduces the coherence matrix, the basis for understanding the concepts involved. Further, it briefly discusses the coherence time and lengths. Section III describes the way the mutual coherence function is propagated through free space and it reminds us of the connection between beam divergence and coherence length. Section IV describes how scattering effects can be incorporated in the coherence theory. Examples are the phase-object approximation and refraction. Section V describes how magnetic scattering can be incorporated by using an extension of the phase-object approximation. Further, it is shown that beam splitting effects due to a magnetic flux density transition can be calculated by the introduced concept. Section VI gives a rigorous derivation of the measured polarization and the so-called shim count rate in a neutron scattering instrument. It shows that these observables can be calculated from the basic principles of the matrix coherence theory introduced here. Section VII gives a derivation of the measured polarization in spin echo small angle neutron scattering instruments for both nonmagnetic and magnetic samples based on the coherence theory and the extension of the phase-object approximation described in Sec. V. Finally, in Sec. VIII, some conclusions are given.

\section{COHERENCE MATRIX THEORY}

To be able to calculate the instrumental effects on the measured count rate using polarized neutrons, one should have a rigorous definition of polarization and measurement methods. For this, the coherence matrix concept is introduced. Although it is not a new concept in coherence theory, ${ }^{1}$ it has not been applied to the propagation of neutrons. Matrix calculations are well known to determine the change in the polarization vector. These calculations are more or less based on the solution of Schrödinger equations for the spinexpectation values. The components of the polarization vector are three parts of the four part Stokes parameters. The fourth part is the beam intensity. The Stokes parameters perfectly describe a quasimonochromatic beam at a certain po- 
sition. They, however, fail to indicate how the polarization or intensity of the beam is propagated. The coherence matrix facilitates this. A further advantage of using the coherence matrix instead of the Stokes parameters is that the relation to the nonmagnetic scattering and the quantum nature of the neutron scattering phenomena is not blurred and that it can be incorporated in coherence theory.

\section{A. Definitions}

For particle wave fields where interaction with magnetic and electric forces cannot be ignored Dirac ${ }^{10}$ was able to reduce the time-dependent Schrödinger equation using two linear dependent solutions. The same procedure is followed to find the equation for neutron wave fields where, in general, electric and gravitational forces are ignored:

$$
\begin{aligned}
i \hbar \frac{\partial}{\partial t}\left(\begin{array}{l}
\Psi^{+}(\vec{r}, t) \\
\Psi^{-}(\vec{r}, t)
\end{array}\right)= & \left\{-\frac{\hbar^{2}}{2 m} \nabla^{2}+V(\vec{r})-g_{r} \mu_{N} \hat{\sigma} \cdot \vec{B}(\vec{r}, t)\right\} \\
& \times\left(\begin{array}{c}
\Psi^{+}(\vec{r}, t) \\
\Psi^{-}(\vec{r}, t)
\end{array}\right),
\end{aligned}
$$

where $\hat{\sigma}$ is the Pauli spin matrix vector [components as shown in Eq. (5)], $V(\vec{r})$ is the complex nuclear optical potential, $g_{r}=-1.9130$ the gyromagnetic ratio, $\mu_{N}=5.051$ $\times 10^{-27} \mathrm{~J} / \mathrm{T}$ the nuclear magneton, and $\vec{B}(\vec{r}, t)$ the magnetic flux density. ${ }^{5}$ The negative gyromagnetic ratio of the neutron represents the fact that the neutron spin is oriented antiparallel to its magnetic moment. The quantity $-g_{r} \mu_{N} B(\vec{r}, t)$ $(\approx 60.3 \mathrm{neV} / \mathrm{T})$ is known as the Zeeman energy as it gives the energy gain (spins parallel to magnetic flux density or "up" spin state) or loss (spins antiparallel to magnetic flux density or "down" spin state) of a neutron experiencing a magnetic flux density. Its value must be compared to the total energy of the neutron given by $\hbar \omega_{k}(\approx 20.5 \mathrm{meV}$ for neutrons with a wavelength of $0.2 \mathrm{~nm}) \cdot \vec{k}$ is the wave vector in vacuum, $\omega_{k}=\hbar k^{2} / 2 m$, and $m$ is the neutron mass. It is assumed that all wave functions are statistically stationary (an ensemble average is independent of the origin of time) and ergodic (an ensemble average is time independent and equal to a time average $)^{1}$ and only second-order coherence effects are discussed. Stationary is related to the frequency $\omega_{k}$ of the neutron wave field. Hence, processes with relevant time constants much larger than $\omega_{k}^{-1}$ can still be treated. Note that $\hbar \omega_{k}$ is determined by the total energy of the neutron (the sum of the kinetic and potential energies) and is constant for a statistically stationary beam.

For moderate values of the magnetic flux density of about $1 / 3 \mathrm{~T}$, the Zeeman energy is 6 orders of magnitude smaller than the kinetic energy. The linear dependent solutions $\Psi^{+}(\vec{r}, t)$ and $\Psi^{-}(\vec{r}, t)$ represent the wave function of, respectively, the up spin state and the down spin state, characterizing the neutron wave field at point $\vec{r}$ at time $t$. For a realistic neutron source, they are fluctuating functions of time and may be regarded as typical members of an ensemble consisting of all possible neutron generating events. They consist of a large number of Fourier components, independent of each other. Their superposition gives rise to a fluctuating field which can only be described in statistical terms. If a uniform, quasimonochromatic, well collimated, and stationary beam is considered, one can define the $2 \times 2$ coherence matrix:

$$
\hat{\Gamma}\left(\vec{r}_{1}, \vec{r}_{2}, \tau\right)=\left\langle( \begin{array} { l } 
{ \Psi ^ { + } ( \vec { r } _ { 2 } , t + \tau ) } \\
{ \Psi ^ { - } ( \vec { r } _ { 2 } , t + \tau ) }
\end{array} ) \left(\begin{array}{ll}
\Psi^{+}\left(\vec{r}_{1}, t\right)^{*} & \left.\left.\Psi^{-}\left(\vec{r}_{1}, t\right)^{*}\right)\right\rangle_{t},
\end{array}\right.\right.
$$

which can be reduced to

$$
\hat{\Gamma}\left(\vec{r}_{1}, \vec{r}_{2}, \tau\right)=\left[\begin{array}{cc}
\Gamma^{++}\left(\vec{r}_{1}, \vec{r}_{2}, \tau\right) & \Gamma^{+-}\left(\vec{r}_{1}, \vec{r}_{2}, \tau\right) \\
\Gamma^{+-}\left(\vec{r}_{2}, \vec{r}_{1},-\tau\right)^{*} & \Gamma^{--}\left(\vec{r}_{1}, \vec{r}_{2}, \tau\right)
\end{array}\right],
$$

where $\Gamma^{++}\left(\vec{r}_{1}, \vec{r}_{2}, \tau\right)$ is the mutual coherence function of the up spin wave, $\Gamma^{--}\left(\vec{r}_{1}, \vec{r}_{2}, \tau\right)$ the same of the down state, and $\Gamma^{+-}\left(\vec{r}_{1}, \vec{r}_{2}, \tau\right)$ the cross coherence function of the two spin states. These functions represent the correlation between the field at a point $P_{1}$ at $\vec{r}_{1}$ and the complex conjugated field at a point $P_{2}$ at $\vec{r}_{2}$ at time moments $t$ and $t+\tau$, respectively. As the considered field is stationary, the ensemble averages are independent of time $t$ and only the time difference between two points influences the mutual coherence. As was already noted by Mezei in $1980,{ }^{9}$ the polarization of a neutron beam can be interpreted as the degree of coherence between the wave functions representing the two spin states of a neutron. Then the local polarization at some position $\vec{r}$ can be described by the elements of the coherence matrix:

$$
P_{j}(\vec{r})=\frac{\operatorname{Tr}\left(\hat{\Gamma}(\vec{r}, \vec{r}, 0) \hat{\sigma}_{j}\right)}{\operatorname{Tr}(\hat{\Gamma}(\vec{r}, \vec{r}, 0))},
$$

where the index $j$ denotes the $x, y$, or $z$ component and $\hat{\sigma}_{j}$ are the Pauli spin matrices:

$$
\hat{\sigma}_{x}=\left(\begin{array}{ll}
0 & 1 \\
1 & 0
\end{array}\right), \quad \hat{\sigma}_{y}=\left(\begin{array}{cc}
0 & -i \\
i & 0
\end{array}\right), \quad \text { and } \hat{\sigma}_{z}=\left(\begin{array}{cc}
1 & 0 \\
0 & -1
\end{array}\right),
$$

where the $z$ axis is the quantization axis. Note that the polarization is fully known if the coherence matrix is known, but not vice versa. Although for insight into the design of an instrument or experiment it can be useful to examine changes in the polarization vector for a neutron passing along a certain path through the instrument, this view is not sufficient to understand all possible effects. For instance, the neutron flux is given by

$$
\vec{J}(\vec{r})=2 \vec{v}_{p} \operatorname{Tr}(\hat{\Gamma}(\vec{r}, \vec{r}, 0)),
$$

where $v_{p}=\hbar \bar{k} / 2 m$ equals the phase velocity of the wave field, half of the neutron velocity, and $\bar{k}$ is the average length of the wave vector $\vec{k}$. Note that this equation holds exactly only for a parallel beam in the direction $\vec{v}_{p}$ (or $\vec{k}$ ). If the divergence of the beam becomes too large, this approximation is not allowed. The time dependence of the density and the neutron flux disappears as only stationary fields are taken into account. Here, it is assumed that the beam has the same direction as $\vec{v}_{p}$ for both up and down spin states. In general, this is not the case as, in principle, the up and down spin states can propagate through a magnetic flux density in different direc- 
tions (see Sec. V B). However, in most practical cases, the coherence length is too small or the spread in directions too large to observe the beam splitting and the above approximation is valid. This was already stressed by Mezei. ${ }^{9,11}$

\section{B. Propagation of coherence matrix}

If the magnetic flux density and complex optical potential are constant in time, the wave equations for the coherence matrix can be derived from their definition [Eq. (2)] and the above Schrödinger equation:

$$
\begin{aligned}
& \nabla_{1}^{2} \hat{\Gamma}\left(\vec{r}_{1}, \vec{r}_{2}, \tau\right)=-\hat{\Gamma}\left(\vec{r}_{1}, \vec{r}_{2}, \tau\right)\left\{k\left(\vec{r}_{1}\right)^{2} \hat{I}+\kappa\left(\vec{r}_{1}\right)^{2}\left[\hat{\sigma} \cdot \vec{n}_{B}\left(\vec{r}_{1}\right)\right]\right\}, \\
& \nabla_{2}^{2} \hat{\Gamma}\left(\vec{r}_{1}, \vec{r}_{2}, \tau\right)=-\left\{k\left(\vec{r}_{2}\right)^{2} \hat{I}+\kappa\left(\vec{r}_{2}\right)^{2}\left[\hat{\sigma} \cdot \vec{n}_{B}\left(\vec{r}_{2}\right)\right]\right\} \hat{\Gamma}\left(\vec{r}_{1}, \vec{r}_{2}, \tau\right),
\end{aligned}
$$

where $\kappa(\vec{r})^{2}$ is a measure for the Zeeman energy in wavevector-squared units $=2 m g_{r} \mu_{N} B(\vec{r}) / \hbar^{2} . \quad k(\vec{r})^{2}=\bar{k}^{2}-2 m V(\vec{r}) /$ $\hbar^{2}$ and $\hat{I}$ is the $2 \times 2$ identity matrix. $\vec{n}_{B}(\vec{r})$ is a unit vector in the direction of the magnetic flux density $\vec{B}(\vec{r})$. These two equations describe the propagation of the coherence matrix and can be solved given a certain profile for the magnetic flux density and complex optical potential. In most cases where the magnetic flux density is generated by macroscopic objects, $\kappa(\vec{r}) \ll k(\vec{r})$, the propagation will be determined mainly by the first term of the above equations and the difference, between the propagation of the elements of the coherence matrix is very small. To underline the difference, let us define the reduced coherence matrix $\hat{\gamma}_{B}$ :

$$
\hat{\Gamma}\left(\vec{r}_{1}, \vec{r}_{2}, \tau\right)=\Gamma_{0}\left(\vec{r}_{1}, \vec{r}_{2}, \tau\right) \hat{\gamma}_{B}\left(\vec{r}_{1}, \vec{r}_{2}, \tau\right),
$$

where $\Gamma_{0}\left(\vec{r}_{1}, \vec{r}_{2}, \tau\right)$ would have been the mutual coherence function of the wave field when all magnetic flux densities were turned off. It obeys the wave equations for the mutual coherence function for the nonmagnetic wave field:

$$
\nabla_{j}^{2} \Gamma_{0}\left(\vec{r}_{1}, \vec{r}_{2}, \tau\right)=-k\left(\vec{r}_{j}\right)^{2} \Gamma_{0}\left(\vec{r}_{1}, \vec{r}_{2}, \tau\right)
$$

for $j=1,2$.

Let the vector $\vec{k}\left(\vec{r}_{1}\right)$ be defined as

$$
\vec{\nabla}_{1} \Gamma_{0}\left(\vec{r}_{1}, \vec{r}_{2}, \tau\right)=-i \vec{k}^{*}\left(\vec{r}_{1}\right) \Gamma_{0}\left(\vec{r}_{1}, \vec{r}_{2}, \tau\right)
$$

The complex conjugate is used because of the definition of $\hat{\Gamma}$ in Eq. (2). Then, also because $\Gamma_{0}\left(\vec{r}_{1}, \vec{r}_{2}, \tau\right)=\Gamma_{0}\left(\vec{r}_{2}, \vec{r}_{1},-\tau\right)^{*}$ :

$$
\vec{\nabla}_{2} \Gamma_{0}\left(\vec{r}_{1}, \vec{r}_{2}, \tau\right)=i \vec{k}\left(\vec{r}_{2}\right) \Gamma_{0}\left(\vec{r}_{1}, \vec{r}_{2}, \tau\right)
$$

For a slowly varying complex optical potential, $\left|\vec{\nabla}_{j} \cdot \vec{k}\left(\vec{r}_{j}\right)\right|$ $\ll\left|k\left(\vec{r}_{1}\right)\right|^{2} \approx\left|k\left(\vec{r}_{2}\right)\right|^{2}$, it can be shown that

$$
\vec{k}\left(\vec{r}_{1}\right)^{*} \cdot \vec{\nabla}_{1} \hat{\gamma}_{B}\left(\vec{r}_{1}, \vec{r}_{2}, \tau\right)=-\frac{i \kappa\left(\vec{r}_{1}\right)^{2}}{2} \hat{\gamma}_{B}\left(\vec{r}_{1}, \vec{r}_{2}, \tau\right)\left[\hat{\sigma} \cdot \vec{n}_{B}\left(\vec{r}_{1}\right)\right],
$$

$$
\vec{k}\left(\vec{r}_{2}\right) \cdot \vec{\nabla}_{2} \hat{\gamma}_{B}\left(\vec{r}_{1}, \vec{r}_{2}, \tau\right)=\frac{i \kappa\left(\vec{r}_{2}\right)^{2}}{2}\left[\hat{\sigma} \cdot \vec{n}_{B}\left(\vec{r}_{2}\right)\right] \hat{\gamma}_{B}\left(\vec{r}_{1}, \vec{r}_{2}, \tau\right) .
$$

Equations (10), (13), and (14) describe the propagation of the coherence matrix. The polarization vector can be calculated directly from the reduced coherence matrix only. The mutual coherence function $\Gamma_{0}$ propagates as if the beam was completely depolarized without interaction with the magnetic flux density. This will be discussed in the next section. Note that it is assumed that the Zeeman energy is much smaller than the kinetic energy of the neutrons. This implies that the reflection or refraction of the neutrons at any transition in the magnetic flux density has been neglected. The differential equations (13) and (14) are not independent of $\Gamma_{0}$ because of the definition (11) of $\vec{k}(\vec{r})$. In the following, the propagation of $\hat{\gamma}_{B}$ is discussed.

\section{Constant magnetic flux density direction}

For some region in space where the direction of the magnetic flux density is constant, one can always make the spinquantization direction equal to this direction by appropriate rotation of the reference frame. ${ }^{12}$ Rotation of the reference frame from the $z$ direction $\left(\vec{e}_{z}\right)$ to the direction of the magnetic flux density,

$$
\vec{n}_{B}=\left(\begin{array}{c}
\cos \vartheta \sin \theta \\
\sin \vartheta \sin \theta \\
\cos \theta
\end{array}\right),
$$

transforms the coherence matrix through

$$
\hat{\Gamma}_{r o t}\left(\vec{r}_{1}, \vec{r}_{2}, \tau\right)=\hat{R}_{\vec{n}_{B}\left(\vec{r}_{2}\right)} \hat{\Gamma}\left(\vec{r}_{1}, \vec{r}_{2}, \tau\right) \hat{R}_{\vec{n}_{B}\left(\vec{r}_{1}\right)}^{\dagger},
$$

where $\hat{R}^{\dagger}$ represents the conjugate transpose of $\hat{R}$ and $\hat{R}_{\vec{n}_{B}}$ $=\hat{T}_{y}(\theta) \hat{T}_{z}(\vartheta)$ and

$$
\hat{T}_{y}(\tau)=\left(\begin{array}{cc}
\cos \frac{\tau}{2} & \sin \frac{\tau}{2} \\
-\sin \frac{\tau}{2} & \cos \frac{\tau}{2}
\end{array}\right)
$$

and

$$
\hat{T}_{z}(\tau)=\left(\begin{array}{cc}
e^{i \tau / 2} & 0 \\
0 & e^{-i \tau / 2}
\end{array}\right) .
$$

For the region of a magnetic flux density in the $z$ or quantization direction, Eqs. (13) and (14) reduce to $\left(\vec{n}_{B}=\vec{e}_{z}\right)$

$$
\begin{aligned}
\vec{k}\left(\vec{r}_{1}\right)^{*} \cdot \vec{\nabla}_{1} \hat{\gamma}_{B}\left(\vec{r}_{1}, \vec{r}_{2}, \tau\right)=-\frac{i \kappa\left(\vec{r}_{1}\right)^{2}}{2} \hat{\gamma}_{B}\left(\vec{r}_{1}, \vec{r}_{2}, \tau\right) \hat{\sigma}_{z}, \\
\vec{k}\left(\vec{r}_{2}\right) \cdot \vec{\nabla}_{2} \hat{\gamma}_{B}\left(\vec{r}_{1}, \vec{r}_{2}, \tau\right)=\frac{i \kappa\left(\vec{r}_{2}\right)^{2}}{2} \hat{\sigma}_{z} \hat{\gamma}_{B}\left(\vec{r}_{1}, \vec{r}_{2}, \tau\right) .
\end{aligned}
$$

For a completely coherent and homogeneous beam in vacuum (a plane wave), $\vec{k}(\vec{r})=\vec{k} \vec{n}_{k}$ is constant and the solution of these differential equations is given by 


$$
\hat{\gamma}_{B}\left(\vec{r}_{1}, \vec{r}_{2}, \tau\right)=\hat{T}_{z}\left(\phi\left(\vec{r}_{2}^{\prime}, \vec{r}_{2}\right)\right) \hat{\gamma}_{B}\left(\vec{r}_{1}^{\prime}, \vec{r}_{2}^{\prime}, \tau\right) \hat{T}_{z}\left(\phi\left(\vec{r}_{1}^{\prime}, \vec{r}_{1}\right)\right)^{\dagger},
$$

where $\vec{r}_{j}-\vec{r}_{j}^{\prime}=\left|\vec{r}_{j}-\vec{r}_{j}^{\prime}\right| \vec{n}_{k}$ and

$$
\phi\left(\overrightarrow{r^{\prime}}, \vec{r}\right)=\bar{k}^{-1} \int_{\vec{r}^{\prime}}^{\vec{r}} \kappa(\vec{r})^{2} d r=\frac{2 m g_{r} \mu_{N}}{\hbar^{2} \bar{k}} \int_{\vec{r}^{\prime}}^{\vec{r}} B(\vec{r}) d r
$$

is a measure for the extra phase acquired by the neutron wave field due to the interaction with the magnetic flux density. Equation (21) shows that the propagation of the coherence matrix from one position to another through a magnetic flux density with constant direction can be calculated by means of the line integral representing the extra phase acquired by a specific neutron (up or down spin state) when traveling from the first position to the last position along the classical neutron path. Combining Eqs. (16) and (21) for a region with constant magnetic flux density direction $\vec{n}_{B}$ results in

$$
\hat{\gamma}_{B}\left(\vec{r}_{1}, \vec{r}_{2}, \tau\right)=\hat{M}\left(\vec{r}_{2}, \vec{r}_{2}^{\prime}\right) \hat{\gamma}_{B}\left(\vec{r}_{1}^{\prime}, \vec{r}_{2}^{\prime}, \tau\right) \hat{M}\left(\vec{r}_{1}, \vec{r}_{1}^{\prime}\right)^{\dagger},
$$

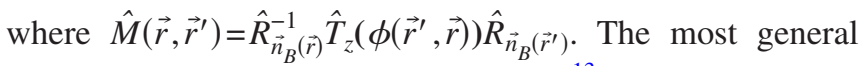
form of this matrix is a rotation matrix. ${ }^{13}$

\section{Larmor precession}

In a region of length $L$ where both the direction, $\vec{n}$ $=\left(n_{x}, n_{y}, n_{z}\right)^{T}$, and the magnitude of the magnetic flux density, $B$, are constant, matrix $\hat{M}$ as defined in the previous section is given by

$$
\hat{M}=\hat{R}_{\vec{n}}^{-1} \hat{T}_{z}(c \bar{\lambda} B L) \hat{R}_{\vec{n}},
$$

where $\quad c=-4 \pi m g_{r} \mu_{N} / h^{2}=4.63209 \times 10^{14} \mathrm{~T}^{-1} \mathrm{~m}^{-2}$. This equation can be rewritten as

$$
\hat{M}=\hat{I} \cos \frac{\gamma_{L} B t_{L}}{2}-i\left(n_{x} \hat{\sigma}_{x}+n_{y} \hat{\sigma}_{y}+n_{z} \hat{\sigma}_{z}\right) \sin \frac{\gamma_{L} B t_{L}}{2},
$$

where $t_{L}=2 L / v_{p}$ is the travel time of a neutron with wavelength $\bar{\lambda}$ through the region of magnetic flux density, $\gamma_{L}$ $=-2 g_{r} \mu_{N} / \hbar=1.832472 \times 10^{8} \mathrm{~T}^{-1} \mathrm{~s}^{-1}$, and $\gamma_{L} B$ is known as the Larmor frequency, independent of the wavelength of the neutrons. This matrix can be interpreted as a rotation of the polarization vector of $\gamma_{L} B t_{L}$ radians around the direction of the magnetic flux density.

\section{E. General magnetic flux density}

If between $\vec{r}$ and $\vec{r}^{\prime}$ the direction of the magnetic flux density changes too much (see Fig. 1), the region between $\vec{r}^{\prime}$ and $\vec{r}$ can be divided into $N$ regions where the direction does not change appreciably. The matrix $\hat{M}\left(\vec{r}, \vec{r}^{\prime}\right)$ in Eq. (23) must be replaced by

$$
\hat{D}\left(\vec{r}, \vec{r}^{\prime}\right)=\prod_{i=1}^{N} \hat{M}_{i}\left(\vec{r}_{i+1}, \vec{r}_{i}\right)
$$

where $\vec{r}_{1}=\vec{r}^{\prime}, \vec{r}_{N+1}=\vec{r}, \vec{r}_{i}$ indicates the border between regions $i$ and $i+1$ and $\hat{M}_{i}\left(\vec{r}_{i+1}, \vec{r}_{i}\right)$ is the matrix describing the propa-

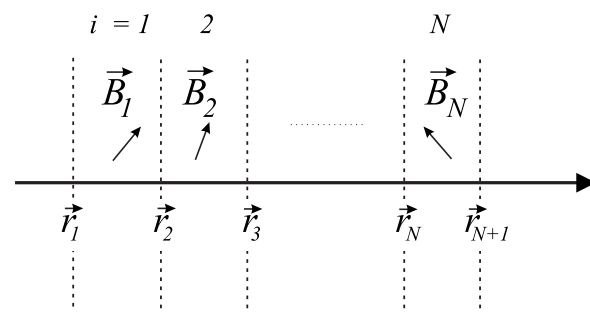

FIG. 1. Sequence in space of magnetic flux densities representing a space dependent magnetic flux density $\vec{B}(\vec{r})$.

gation of the reduced coherence matrix in region $i$. Note that as $\hat{D}$ is a product of rotation (or streaming) matrices only, it can also be represented by a rotation matrix. For a constant magnetic flux density and optical potential, the propagation of the coherence matrix can also be derived in the way shown by Mandel and Wolf. ${ }^{1}$ The derivation uses the Rayleigh diffraction formula of the first kind. The Rayleigh diffraction formula just describes the wave function in the halfspace $x>0$ given its value and derivative at $x=0$. Hence, it does not really describe a propagation but it is just a solution of the wave equation with the correct boundary conditions. However, within certain limits, the solution of a wave equation can be understood as the propagation of a ray. ${ }^{5}$ The propagation of the coherence matrix can be derived by applying the definition of the coherence matrix and using the Rayleigh diffraction formula twice for each component of the matrix separately. Assuming that the wave field is quasimonochromatic, the propagation of the coherence matrix is given by

$$
\begin{aligned}
\hat{\Gamma}\left(\vec{r}_{1}, \vec{r}_{2}, \tau\right)= & \iint_{x=0} \cos \theta_{1} \cos \theta_{2} \hat{T}_{k}\left(R_{2}\right) \hat{\Gamma}\left(\overrightarrow{r_{1}^{\prime}}, \vec{r}_{2}^{\prime}, \tau\right) \\
& \times \hat{T}_{k}\left(R_{1}\right)^{\dagger} d^{2} r_{1}^{\prime} d^{2} r_{2}^{\prime},
\end{aligned}
$$

where

$$
\hat{T}_{k}(R)=\frac{1}{2 \pi R}\left(\begin{array}{cc}
k^{+} e^{i k^{+} R} & 0 \\
0 & k^{-} e^{i k^{-} R}
\end{array}\right)
$$

and $k^{ \pm}=\left(\bar{k}^{2} \pm \kappa^{2}\right)^{1 / 2} \cdot k^{+}$represents the (average) wave vector of the up spin wave (parallel to the magnetic flux density) and $k^{-}$the same for the down spin wave. The integrals are taken over points $S_{1}$ at $\vec{r}_{1}^{\prime}$ and $S_{2}$ at $\vec{r}_{2}^{\prime}$ of plane $x=0, \vec{R}_{i}=\vec{r}_{i}$ $-\vec{r}_{i}^{\prime}$, and $\theta_{i}$ is the angle between the line $P_{i} S_{i}$ and the $x$ axis (see also Fig. 4.9 in the textbook by Mandel and Wolf ${ }^{1}$ ). It was assumed that $R_{i} \gg 2 \pi / \bar{k}$, corresponding to ignoring the evanescent waves. Because $\kappa \ll \bar{k}$, it is possible to expand $k^{ \pm}$ as $\bar{k} \pm \delta \kappa$, where $\delta \kappa=\kappa^{2} / 2 \bar{k}$, and use the approximation

$$
\hat{T}_{k}(R) \approx \frac{\bar{k}}{2 \pi R} e^{i \bar{k} R} \hat{T}_{z}(\delta \kappa R)
$$

Note that the elements of this matrix resemble Green functions, describing the propagation of a converging spherical wave field toward the origin at $r=0$ in each half-space $(x$ $>0$ and $x<0$ ). The complex conjugated Green's function 
describes a diverging spherical wave field from the origin. One should realize that the propagated coherence matrix is calculated by two integrals. One can be interpreted as the propagation of the incident wave function reaching point $\vec{r}_{1}$ integrated over the source wave function at $\vec{r}_{1}^{\prime}$. The other integral does the same for the incident wave function reaching point $\vec{r}_{2}$ from $\vec{r}_{2}^{\prime}$. The cosine factors represent the fact that the solid angle in the field of vision of the observer of a surface area when seen under an angle scales with the cosine of that angle. In most cases of practical interest, the points $P_{1}$ and $P_{2}$ are situated in the far zone of the source. Then, $\cos \theta_{j}=\frac{\vec{R}_{j} \cdot \vec{e}_{x}}{R_{j}}$, where $\vec{e}_{x}$ is the unit vector in the $x$ direction. In the far zone, $\cos \theta_{1} \approx \cos \theta_{2}, R_{j} \approx r_{j}-\frac{\vec{r}_{j} \cdot \vec{r}_{j}^{\prime}}{r_{j}}$, so that $R_{2}-R_{1}$ $=r_{2}-r_{1}-r_{12}$ where $r_{12}=\left(\frac{\vec{r}_{2} \cdot \vec{r}_{2}^{\prime}}{r_{2}}-\frac{\vec{r}_{1} \cdot \vec{r}_{1}^{\prime}}{r_{1}}\right)$. Also, $R_{j}$ can be replaced by $r_{j}$ in the cosine factor and in the denominator of Eq. (28).

If the transversal coherence length (see also next section) of the neutron beam, $r_{c} \ll \delta \kappa^{-1}$, and the far zone of the field is considered, the matrices just before and after the coherence matrix inside the integral do not depend on $\vec{r}_{1}^{\prime}$ and $\vec{r}_{2}^{\prime}$ and can be taken outside the integral. It can be repeated over subsequent regions in space where a (different strength) magnetic flux density exists, so that the arguments of the matrices before and after the integral are converted into a sum over all regions which is equal to the line integral as defined in equation (22). The above equation reduces to Eq. (21) if $\hat{\gamma}_{B}\left(\vec{r}_{1}^{\prime}, \vec{r}_{2}^{\prime}, \tau\right)$ in the $y z$ plane at $x=0$ does not depend on $\vec{r}_{1}^{\prime}$ or $\vec{r}_{2}^{\prime}$. One can extend the above reasoning to the general case where the magnetic flux density varies slowly in space. Slowly means that the changes in the magnetic flux density are on a scale much larger than $\delta \kappa^{-1}$. In this situation, the matrices before and after the coherence matrix in Eq. (27) are converted into matrices according to Eq. (26) and Eq. (27) reduces in the far-zone limit to

$$
\begin{aligned}
\hat{\Gamma}\left(\vec{r}_{1}, \vec{r}_{2}, \tau\right)= & \frac{\cos \theta_{1} \cos \theta_{2} e^{i k\left(r_{2}-r_{1}\right)}}{r_{1} r_{2} \bar{\lambda}^{2}} \iint_{x=0} e^{-i \bar{k} r_{12}} \hat{D}\left(\vec{r}_{2}, \vec{r}_{2}^{\prime}\right) \\
& \times \hat{\Gamma}\left(\vec{r}_{1}^{\prime}, \vec{r}_{2}^{\prime}, \tau\right) \hat{D}\left(\vec{r}_{1}, \vec{r}_{1}^{\prime}\right)^{\dagger} d^{2} r_{1}^{\prime} d^{2} r_{2}^{\prime},
\end{aligned}
$$

where $r_{12}=\frac{\vec{r}_{2} \cdot \vec{r}_{2}^{\prime}}{r_{2}}-\frac{\vec{r}_{1} \cdot \vec{r}_{1}^{\prime}}{r_{1}}$. It is also possible to calculate matrix $\hat{D}$ if the magnetic flux density changes in a specific way. This was done by Schwink and Schärpf ${ }^{14}$ for helical magnetic structures. They found that in these cases, the up and down spin states propagate both with the two wave vectors $\vec{k}^{+}$and $\vec{k}^{-}$. The propagation reduces to a propagation with one wave vector if the quantization axis is continuously rotated and taken along the direction of the magnetic flux density.

\section{F. Coherence time and lengths}

The coherence time $t_{c}$ of a wave field is a measure of the time interval in which appreciable amplitude and wave correlations of the wave field at a particular point $P$ in a fluctuating field will persist. The mutual coherence function can be used to define the coherence time of a wave field. This has been discussed by Mandel and Wolf. ${ }^{1}$ For a quasimonochromatic beam (when the wave field is quasimonochromatic and the spectrum has a single reasonably well-defined peak), the coherence time is related to the effective spectral width of the wave field at point $P$. In nondispersive media (and stationary fields), the temporal coherence can be expressed in terms of the spatial coherence. Under such conditions, the coherence time is related to the longitudinal coherence length $l_{c}$, which is the distance traveled by the wave field during the coherence time. As the neutron phase velocity depends on the neutron wavelength, this limits the applicability of general coherence theory to quasimonochromatic beams. For a quasimonochromatic wave field, the phase velocity can be taken constant, so the longitudinal coherence length is given by

$$
l_{c}=v_{p} t_{c} \geqslant \frac{1}{2 \Delta k}=\frac{\bar{\lambda}^{2}}{4 \pi \Delta \lambda},
$$

where $\bar{\lambda}$ is the average wavelength of the wave field and $\Delta \lambda$ the effective wavelength spread equal to $\Delta k \bar{\lambda}^{2} / 2 \pi$. The general coherence length $r_{c}$ is defined in a similar way as the coherence time: the distance at which appreciable amplitude and wave correlations of the wave field in a fluctuating field will persist. Note that the term longitudinal applies to the direction of the propagation of the wave field. Only if the wave field can be regarded as a beam does the term become useful. However, one must keep in mind the conceptual difference between the longitudinal coherence length as derived from the time dependence of the mutual coherence function and the general coherence length as derived from the spatial dependence of the mutual coherence function. In a directed quasimonochromatic stationary wave field, the longitudinal coherence length is the same as the general coherence length taken along the beam direction. Then, the general coherence lengths in directions perpendicular to the beam are referred to as transversal coherence lengths.

\section{PROPAGATION OF MUTUAL COHERENCE FUNCTION}

As was discussed in the previous section, under certain conditions, the propagation of the coherence matrix can be split into the propagation of the mutual coherence function representing an unpolarized beam and the reduced coherence matrix representing the polarization properties. The propagation of the mutual coherence function can be split into three parts: propagation from source to sample, propagation through the sample, and propagation from sample to detector. The propagation from source to sample and sample to detector is assumed to be through vacuum.

\section{A. Propagation in free space}

For an unpolarized beam in free space $[V(\vec{r})=0]$ with the magnetic interactions neglected, the matrix elements in Eqs. (7) and (8) become independent. Then a matrix element can be interpreted as the mutual coherence function. This function can propagate into the half-space $x>0$ yielding the well known coherence propagation law. Here, this law follows directly from Eq. (27) where the matrices are reduced to simple functions: 


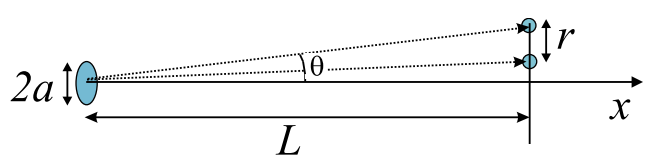

FIG. 2. (Color online) Notation relating to the propagation of the mutual coherence function from source to a plane at a distance $L$ from the source.

$$
\begin{aligned}
& \Gamma_{0}\left(\vec{r}_{1}, \vec{r}_{2}, \tau\right) \\
& \quad=\iint_{x=0} \frac{\cos \theta_{1} \cos \theta_{2} e^{i k\left(R_{2}-R_{1}\right)}}{\bar{\lambda}^{2} R_{1} R_{2}} \Gamma_{0}\left(\overrightarrow{r_{1}^{\prime}}, \vec{r}_{2}^{\prime}, \tau\right) d^{2} r_{1}^{\prime} d^{2} r_{2}^{\prime} .
\end{aligned}
$$

This equation enables the calculation of the propagation of the mutual coherence function from neutron source to sample and from sample to detector.

\section{B. Propagation from source to sample}

A typical neutron source consists of unrelated neutron generating events and, limiting the case to stationary phenomena, the mutual coherence function at the source position $(x=0)$ is given by ${ }^{1}$

$$
\Gamma\left(\overrightarrow{r_{1}^{\prime}}, \overrightarrow{r_{2}^{\prime}}, \tau\right)=\frac{1}{2 v_{p}} \frac{J_{0}\left(\vec{r}_{1}^{\prime}\right)}{4 \pi} \bar{\lambda}^{2} \delta^{(2)}\left(\overrightarrow{r_{1}^{\prime}}-\overrightarrow{r_{2}^{\prime}}\right) e^{-i \bar{k} v_{p} \tau},
$$

where $J_{0}(\vec{r})$ is the isotropic neutron source flux in neutrons per second per meter squared at $\vec{r} . \delta^{(2)}$ is the two-dimensional Dirac delta function, expressing the mutually uncorrelated source elements. The normalization factors are introduced to transform the neutron flux to the density of the wave field. Although this is an idealization, it will hold if the correlations extend only over distances of the same order as the neutron wavelength and the source dimensions are much larger. Substituting this in Eq. (32) yields for the mutual coherence function

$$
\begin{aligned}
\Gamma\left(\vec{r}_{1}, \vec{r}_{2}, \tau\right)= & \frac{1}{2 v_{p}} \frac{e^{i \bar{k}\left(r_{2}-r_{1}-v_{p} \tau\right)} \cos \theta_{1} \cos \theta_{2}}{4 \pi r_{1} r_{2}} \\
& \times \int_{x=0} e^{-i \vec{q}_{12} \cdot \vec{r}^{\prime}} J_{0}\left(\vec{r}^{\prime}\right) d^{2} r^{\prime},
\end{aligned}
$$

where $\vec{q}_{12}=\bar{k}\left(\vec{r}_{2} / r_{2}-\vec{r}_{1} / r_{1}\right)$. This equation can be used to calculate the transversal coherence length at a distance $L$ of a circular homogeneous completely incoherent quasimonochromatic source with radius $a$ with its surface perpendicular to the $x$ axis (see also Fig. 2). Then, $\vec{r}_{1}=\left(L, y_{1}, z_{1}\right)^{T}, \vec{r}_{2}$ $=\left(L, y_{2}, z_{2}\right)^{T}$, and the mutual coherence function becomes

$$
\Gamma\left(\vec{r}_{1}, \vec{r}_{2}, \tau\right)=\frac{J_{0} a^{2}}{4 v_{p} r_{1} r_{2}} e^{i \bar{k}\left(r_{2}-r_{1}-v_{p} \tau\right)} \frac{J_{1}(\nu)}{\nu},
$$

where $\nu=\bar{k} a r / L, \quad r=\sqrt{\left(y_{2}-y_{1}\right)^{2}+\left(z_{2}-z_{1}\right)^{2}}$ the distance between the two points, and $J_{1}(\nu)$ the first order Bessel function. If the coherence length is defined as

$$
r_{c}(\vec{r})=\frac{\int_{-\infty}^{\infty}\left|r_{1}\right|\left|\Gamma\left(\vec{r}, \vec{r}+\vec{r}_{1}, 0\right)\right|^{2} d r_{1}}{\int_{-\infty}^{\infty}\left|\Gamma\left(\vec{r}, \vec{r}+\vec{r}_{1}, 0\right)\right|^{2} d r_{1}},
$$

this yields

$$
r_{c}=\frac{L}{\bar{k} a} \frac{\int_{0}^{\infty} u^{-1} J_{1}(u)^{2} d u}{\int_{0}^{\infty} u^{-2} J_{1}(u)^{2} d u},
$$

so that

$$
r_{c}=\frac{L}{\bar{k} a} \frac{3 \pi}{8} \approx 1.178 \frac{L}{\bar{k} a} .
$$

If the source area is $1 \mathrm{~mm}^{2}$ and the distance is $4 \mathrm{~m}$, then the coherence length for $0.2 \mathrm{~nm}$ neutrons is about $250 \mathrm{~nm}$. Sometimes, the transversal coherence length is defined as the distance over which the modulus of the normalized mutual coherence function is reduced from its maximum value of 1 for $r=0$ to 0.88 at $r=r_{c}$. This value is reached for $\nu=1$; hence, $r_{c}=L / \bar{k} a$. Another possibility is to use the first zero of $J_{1}$ for $\nu=3.83$ as a measure for the coherence length. All definitions are a bit different from each other but clearly have the same order of magnitude.

\section{Propagation from sample to detector}

In the detector, the count rate (assuming 100\% detector efficiency) is determined as an integral of the neutron flux $\vec{J}\left(\vec{r}_{d}\right)$ over the detector area $A_{d}$ at a position $\vec{r}_{d}$ :

$$
I_{d}=\int_{A_{d}} \vec{J}\left(\overrightarrow{r_{d}}\right) \cdot \vec{n}_{A_{d}} d^{2} r_{d}
$$

where $\vec{n}_{A_{d}}$ is a unit vector perpendicular to the detector area. Using Eq. (6) and assuming $\vec{v}_{p} \cdot \vec{n}_{A_{d}}=v_{p}$, this reduces to

$$
I_{d}=2 v_{p} \int_{A_{d}} \Gamma\left(\overrightarrow{r_{d}}, \overrightarrow{r_{d}}, 0\right) d^{2} r_{d}
$$

Assuming that the whole sample is in the half-space $x<0$, then the mutual coherence function at $x=0$ is propagated to the detector. One can use Eq. (32) to calculate $\Gamma\left(\vec{r}_{d}, \vec{r}_{d}, 0\right)$ :

$$
\Gamma\left(\overrightarrow{r_{d}}, \overrightarrow{r_{d}}, 0\right)=\frac{\cos ^{2} \theta_{d}}{r_{d}^{2} \bar{\lambda}^{2}} \iint_{x=0} e^{-i \vec{p} \cdot\left(\overrightarrow{r_{2}^{\prime}}-\overrightarrow{r_{1}^{\prime}}\right)} \Gamma\left(\overrightarrow{r_{1}^{\prime}}, \overrightarrow{r_{2}^{\prime}}, 0\right) d^{2} r_{1}^{\prime} d^{2} r_{2}^{\prime},
$$

where $\vec{p}=\vec{k} \vec{r}_{d} / r_{d}$. Here, the detector is in the far zone of the sample. If the spatial coherence is much smaller than the sample area, Eq. (41) can be reduced to

$$
\Gamma\left(\overrightarrow{r_{d}}, \overrightarrow{r_{d}}, 0\right)=\frac{\cos ^{2} \theta_{d}}{r_{d}^{2}} \int_{x=0} e^{-i \vec{p} \cdot \overrightarrow{r^{\prime}}} F\left(\overrightarrow{r^{\prime}}\right) d^{2} r^{\prime},
$$

where 


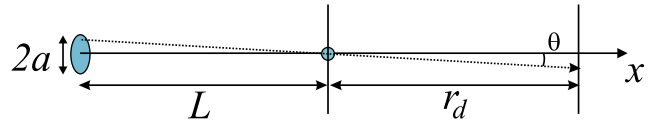

FIG. 3. (Color online) Notation relating to the propagation of the mutual coherence function from a source through a pinhole at the sample position toward a virtual detector at a distance $r_{d}$ from the sample.

$$
F\left(\vec{r}^{\prime}\right)=\bar{\lambda}^{-2} \int_{x=0} \Gamma\left(\vec{r}_{1}^{\prime}, \vec{r}^{\prime}+\vec{r}_{1}^{\prime}, 0\right) d^{2} r_{1}^{\prime}
$$

represents the static mutual coherence function averaged over the beam cross section at the sample position directly after the sample. If the detector area is small and $\cos \theta_{d}$ and $\vec{r}_{d}$ can be taken constant, the detector count rate is

$$
I_{d}\left(\vec{r}_{d}\right)=2 v_{p} \frac{A_{d} \cos ^{2} \theta_{d}}{r_{d}^{2}} \int_{x=0} e^{-i \vec{p} \cdot \overrightarrow{r^{\prime}}} F\left(\overrightarrow{r^{\prime}}\right) d^{2} r^{\prime} .
$$

This equation describes the detector count rate as a twodimensional Fourier transform of the (static) mutual coherence function at $x=0$ integrated over the $y z$ plane or beam cross section at the sample position directly after the sample.

\section{Propagation from source to detector without sample}

For a good understanding, the count rate in the detector can be determined assuming that the sample is the neutron source. In this case, the mutual coherence function, $\Gamma\left(\vec{r}_{1}, \vec{r}_{2}, \tau\right)$, at the sample position according to Eq. (33) can be used in Eq. (41) [or Eq. (34) can be used with Eq. (40)] so that

$$
I_{d}=\int_{A_{d}} \frac{\cos ^{2} \theta_{d}}{4 \pi r_{d}^{2}} d^{2} r_{d} \int_{x=0} J_{0}\left(\vec{r}_{1}^{\prime}\right) d^{2} r_{1}^{\prime} .
$$

If the detector area is small, so that $r_{d}$ and $\cos \theta_{d}$ can be taken constant, this reduces to

$$
I_{d}=\frac{A_{d} \cos ^{2} \theta_{d}}{4 \pi r_{d}^{2}} \int_{x=0} J_{0}\left(\overrightarrow{r_{1}^{\prime}}\right) d^{2} r_{1}^{\prime} .
$$

If the source is homogeneous and limited to an area $A_{0}$ :

$$
I_{d}=J_{0} A_{0} \frac{A_{d} \cos ^{2} \theta_{d}}{4 \pi r_{d}^{2}} .
$$

\section{E. Beam divergence}

The relation between beam divergence and the mutual coherence function can be underlined using the results of the previous sections. Assume the beam to be created from an incoherent source (with area $A_{0}$, isotropic neutron flux $J_{0}$, and position $x=-L$ ), as described by Eq. (33). The beam is directed toward a pinhole (with area $A_{s}$ ) at the sample position. The beam is virtually detected at position $x=r_{d}$ after the pinhole (see Fig. 3). Assume that at $x=0$, the mutual coher- ence function is given by Eq. (35); then, $\Gamma\left(\vec{r}_{d}, \vec{r}_{d}, 0\right)$ is given by

$$
\Gamma\left(\vec{r}_{d}, \vec{r}_{d}, 0\right)=\frac{J_{0} A_{0} A_{s} r_{c}^{2}}{8 v_{p} r_{d}^{2} L^{2} \bar{\lambda}^{-2}} \int_{0}^{\infty} e^{-i q_{r} r_{c} \nu} J_{1}(\nu) d \nu
$$

where $q_{r}$ is the radial component of $\vec{p}$ and $r_{c}=L / \bar{k} a$, the transversal coherence length at $x=0$ due to an incoherent source with radius $a$ at position $x=-L$. For the divergence of the beam at $x=r_{d}$, the spread in angles, $\Delta \theta$, is used, defined as

$$
\begin{aligned}
\Delta \theta^{2}= & \left\langle\theta^{2}\right\rangle-\langle\theta\rangle^{2}=\frac{\int_{A_{d}} \theta^{2} \Gamma\left(\vec{r}_{d}, \vec{r}_{d}, 0\right) d^{2} r_{d}}{\int_{A_{d}} \Gamma\left(\vec{r}_{d}, \vec{r}_{d}, 0\right) d^{2} r_{d}} \\
& -\left(\frac{\int_{A_{d}} \theta \Gamma\left(\vec{r}_{d}, \vec{r}_{d}, 0\right) d^{2} r_{d}}{\int_{A_{d}} \Gamma\left(\vec{r}_{d}, \vec{r}_{d}, 0\right) d^{2} r_{d}}\right)^{2},
\end{aligned}
$$

where the beam propagates along the $x$ axis and the angles are assumed to be small compared to 1 (Fig. 3). Note that $\int_{A_{d}} \Gamma\left(\vec{r}_{d}, \vec{r}_{d}, 0\right) d^{2} r_{d}$ is proportional to the total number of neutrons per second moving through the sample aperture. As $\theta$ $=q_{r} / \bar{k}$ and $\langle\theta\rangle=0$, Eq. (49) becomes

$$
\Delta \theta^{2}=\frac{1}{\bar{k}^{2} r_{c}^{2}} \frac{\int_{0}^{\infty} \eta^{2} \int_{0}^{\infty} e^{-i \eta \nu} J_{1}(\nu) d \nu d \eta}{\int_{0}^{\infty} \int_{0}^{\infty} e^{-i \eta \nu} J_{1}(\nu) d \nu d \eta},
$$

or

$$
\Delta \theta=\frac{1}{\bar{k} r_{c}} \approx \frac{a}{L} .
$$

The approximation at the right hand side follows from Eq. (38). Hence, the beam divergence is inversely proportional to the wave vector and the coherence length and in this case determined by the source dimensions and the distance of the aperture (or pinhole) to the source. This underlines the connection between the coherence theory and the classical raytracing method. In this simple derivation, it was assumed that the pinhole was much larger than the neutron wavelength; hence, no Fresnel diffraction occurs and the Eikonal approximation is valid. ${ }^{5}$

\section{SMALL ANGLE SCATTERING}

The propagation in free space was elaborately discussed in the previous section. For the propagation through a sample, the standard solutions of the Schrödinger equation are used. By applying the definition of the mutual coherence function, these solutions can be transformed to a form where the mutual coherence function, directly after the sample is expressed in a sample correlation function and the mutual coherence function just before the sample.

\section{A. Phase-object approximation}

de Haan et $a l .{ }^{8}$ showed that for small angle neutron scattering and thin samples, it is possible to directly relate the mutual coherence function behind the sample to the one be- 
fore the sample using Feynman path integrals. ${ }^{15}$ In the small angle approximation, the wave function can be calculated by estimating the phase acquired by the neutron wave as if it had followed the classical path through the sample. Hence, the wave function behind the sample becomes

$$
\Psi_{s c}\left(x_{1}, y, z\right)=\Psi_{i n}\left(x_{0}, y, z\right) \exp \left[i \bar{k} \int_{x_{0}}^{x_{1}} n(x, y, z) d x\right],
$$

where $x$ is the incident beam direction, $x=x_{0}$ a plane just before the sample, $x=x_{1}$ a plane just behind the sample, and $n(x, y, z)$ the local refraction index of the sample. The integral in the exponent is known as the optical path length. As it is assumed that the scattering object only influences the phase of the neutron wave function, this is called the phaseobject approximation. This approximation can also be derived using the Eikonal approximation ${ }^{5,16}$ and holds for $|\nabla V(\vec{r})| \ll|\bar{k} V(\vec{r})|$, which is valid as long as two conditions are fulfilled. ${ }^{17}$ First, the difference in optical path length between two straight lines through the sample with a small angle $(2 \theta)$ between them should be much smaller than the neutron wavelength; hence, $\left(x_{1}-x_{0}\right) \theta^{2} \ll \bar{\lambda} / 2$ or $x_{1}-x_{0} \ll 8 \pi^{2} / q^{2} \bar{\lambda}$, where $q=2 \theta \bar{k}$. For instance, if $q \leqslant 0.01 \mathrm{~nm}^{-1}$ and $\bar{\lambda}$ $\approx 0.2 \mathrm{~nm}$, then $x_{1}-x_{0} \ll 4 \mathrm{~mm}$. Second, the local refractive index should not change appreciably from one path to the other. If these conditions are not satisfied, it is possible to make a second-order correction or to break up the calculation in slices of small enough pieces. ${ }^{17}$

The refraction index is related to the potential $V(x, y, z)$ given by the Fermi pseudopotential:

$$
n^{2}=1-\frac{V(x, y, z)}{\hbar \omega_{k}}=1-\frac{\lambda^{2} \rho_{b}(x, y, z)}{\pi}=1-\frac{\lambda^{2} k_{c}^{2}}{4 \pi^{2}},
$$

where $\hbar \omega_{k}$ equals the total energy of the neutron, $\lambda$ its wavelength, $\rho_{b}(x, y, z)$ the coherent scattering length density, and $k_{c}$ the critical wave vector defined by $\sqrt{4 \pi \rho_{b}}$. Incoherent scattering, large angle coherent scattering, or absorption can be taken into account by a suitable imaginary part of the refraction index. ${ }^{5}$ The approximation is valid for thermal neutrons as used in spin echo small angle neutron scattering ${ }^{18-21}$ (SESANS) or ultrasmall angle neutron scattering ${ }^{22-24}$ as the refractive index is close to unity. Note that the deviation from unity of the refractive index is proportional to the square of the wavelength so it increases rapidly for increasing wavelength.

Propagation of the mutual coherence function from $x=x_{0}$ to $x=x_{1}$ (through the sample) can be calculated using Eq. (52) and the definition of the mutual coherence function:

$$
\Gamma_{s c}\left(\vec{r}_{1}, \vec{r}_{2}, \tau\right)=\Gamma_{i n}\left(\vec{r}_{1}-\vec{d}_{x}, \vec{r}_{2}-\vec{d}_{x}, \tau\right) e^{i S\left(\vec{r}_{2}-\vec{d}_{x}\right)-i S^{*}\left(\vec{r}_{1}-\vec{d}_{x}\right)},
$$

where $\vec{d}_{x}=\left(x_{1}-x_{0}\right) \vec{e}_{x}$ and

$$
S(\vec{r})=\bar{k} \int_{0}^{d_{x}}\left(n\left(\vec{r}+x \vec{e}_{x}\right)-1\right) d x
$$

is the extra phase shift acquired by the neutron wave function due to the material properties of the sample. Note that $S$ can be complex due to a complex refraction index. In principle, the subtraction of 1 from the refractive index is not needed and it has no influence on the results. However, because the refractive index only differs from 1 by a small amount, the extra acquired phase shift is much smaller than the total phase shift acquired after traveling through the sample. The use of the extra acquired phase shift enables series expansion of the equation for small values of $S$.

These equations can be used together with Eq. (44) to determine the count rate at the detector. By realizing that $\cos \theta_{d} \approx 1$ for small angle neutron scattering, the result is

$$
I_{d}=2 v_{p} \frac{A_{d}}{r_{d}^{2}} \int_{x=x_{1}} e^{-i \vec{p} \cdot \vec{r}} F(\vec{r}) d^{2} r
$$

where

$$
F(\vec{r})=\bar{\lambda}^{-2} \int_{x=x_{0}} \Gamma_{i n}\left(\vec{r}_{1}, \vec{r}+\vec{r}_{1}, 0\right) e^{i S\left(\vec{r}+\vec{r}_{1}\right)-i S^{*}\left(\vec{r}_{1}\right)} d^{2} r_{1} .
$$

An important feature of this equation is that it describes what normally in SANS is called the scattered neutron intensity and the direct beam. In the above formalism, there is no difference any more: the direct beam is also refracted or in other words, there is no direct beam. Another important feature is that, in general, the coherence function (54) does not have to be a real valued function so that the scattering profile can be different for positive and negative wave vectors.

Assume that the incident beam has a homogeneous intensity over the sample cross section; then, Eq. (56) reduces to

$$
I_{d}=\frac{2 v_{p} A_{d}}{r_{d}^{2} \lambda^{2}} \int_{x=x_{1}} G_{r}(\vec{r}) e^{-i \vec{p} \cdot \vec{r}} \Gamma_{i n}\left(\vec{r}_{s 0}, \vec{r}+\vec{r}_{s 0}, 0\right) d^{2} r,
$$

where $G_{r}(\vec{r})$ is the sample correlation function:

$$
G_{r}(\vec{r})=\int_{x=x_{0}} e^{i S\left(\vec{r}+\vec{r}_{1}\right)-i S^{*}\left(\vec{r}_{1}\right)} d^{2} r_{1} .
$$

Equation (58) can be rewritten as a convolution of the instrumental resolution and the two-dimensional Fourier transform of the sample correlation function:

$$
I_{d}=R^{F T}(\vec{q}) * S_{k}(\vec{q}),
$$

where $\vec{q}=\vec{p}-\vec{k}$. The instrumental resolution is given by

$$
R^{F T}(\vec{q})=\frac{J_{0} A_{d}}{r_{d}^{2} \lambda^{2}} \int_{x=x_{1}} \int_{A_{0}} \frac{e^{-i \vec{q} \cdot \vec{r}}}{4 \pi\left|\vec{r}_{s 0}-\vec{r}^{\prime}\right|^{2}} d^{2} r^{\prime} d^{2} r
$$

and

$$
S_{k}(\vec{q})=\int_{x=x_{1}} e^{-i \vec{q} \cdot \vec{r}} G_{r}(\vec{r}) d^{2} r .
$$

\section{B. Refraction}

Refraction and diffraction occur when a neutron beam travels through a medium where the optical potential of the medium depends on the position. In general, diffraction can be seen as an interference phenomenon of coherent parts of 


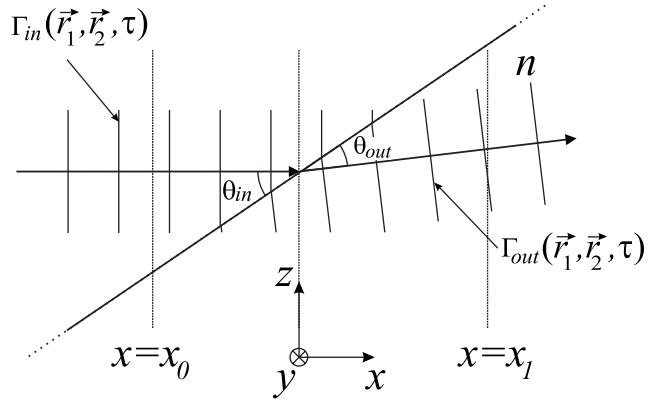

FIG. 4. Refraction geometry projected on a plane with constant $y$.

the scattered beam. Refraction can be understood as the interference phenomenon of the coherent direct beam. However, as was shown in the previous section, the differentiation in direct and scattered beams is somewhat arbitrary. Here, refraction is defined as small angle scattering at macroscopic surfaces. An example of refraction is shown in Fig. 4. Let space be divided into two half-spaces with a boundary making an angle of $\theta_{i n}$ with the incident neutron beam parallel to the $x$ axis. The refractive index of the left half-space is 1 and that of the right is $n$. Assume that a neutron wave function, described by the mutual coherence function $\Gamma_{i n}$, is known at a $y z$ plane at $x=x_{0}$, and the $y$ dependence is ignored. The mutual coherence function at the position $x=x_{1}$ can be calculated using the results of Eqs. (54) and (55). The propagation of the mutual coherence function from $x=x_{1}$ to the detector position is given by Eq. (60). Ignoring resolution effects and absorption or scattering (the refractive index of the medium is real), the count rate in the detector position is proportional to $S_{k}$, which in this case is given by

$$
S_{k}(\vec{q})=\int_{x=x_{0}} \int_{x=x_{1}} e^{-i \vec{q} \cdot \vec{r}_{1}} e^{i S\left(\vec{r}_{1}+\vec{r}_{0}\right)-i S^{*}\left(\vec{r}_{0}\right)} d^{2} r_{1} d^{2} r_{0},
$$

where

$$
S\left(\vec{r}_{1}+\vec{r}_{0}\right)-S^{*}\left(\vec{r}_{0}\right)=\bar{k}(1-n) \cot \theta_{i n}\left(\vec{r}_{1} \cdot \vec{e}_{z}\right)
$$

only depends on $\vec{r}_{1}$, so that Eq. (63) reduces to

$$
S_{k}(\vec{q})=\int_{x=x_{0}} \int e^{i\left\langle\bar{k}(1-n) \cot \theta_{i n}\left(\vec{r}_{1} \cdot \vec{e}_{z}\right)-\vec{q} \cdot \vec{r}_{1}\right\}} d^{2} r_{1} d^{2} r_{0} .
$$

The inner integral of this equation is taken over the whole $x=x_{1}$ plane and only gives a nonzero result when the exponent is 0 . In this case, $\vec{q}=\vec{k} \vec{r}_{d} / r_{d}$, where $\vec{r}_{d}$ is the detector position. Hence,

$$
\left[(1-n) \cot \theta_{i n} \vec{e}_{z}-\vec{r}_{d} / r_{d}\right] \cdot \vec{r}_{1}=0,
$$

where it was used that the $y$ component of $\vec{r}_{d}$ is zero, the $z$ component is $r_{d} \sin \left(\theta_{\text {in }}-\theta_{\text {out }}\right)$, and the $x$ component is $r_{d} \cos \left(\theta_{\text {in }}-\theta_{\text {out }}\right) . \theta_{\text {out }}$ is the refraction angle and is determined by the direction in which the detector is seen at the sample position. Realizing that $\vec{r}_{1}$ has no $y$ component, this reduces to

$$
(1-n) \cot \theta_{\text {in }}=\sin \left(\theta_{\text {in }}-\theta_{\text {out }}\right) \text {. }
$$

If both sides of this equation are multiplied by $\sin \theta_{\text {in }}$ and then to both sides $\cos \theta_{\text {in }} \cos \left(\theta_{\text {in }}-\theta_{\text {out }}\right)$ is added, this becomes

$$
\left[1-n+\cos \left(\theta_{\text {in }}-\theta_{\text {out }}\right)\right] \cos \theta_{\text {in }}=\cos \theta_{\text {out }},
$$

which under the condition that $\left|\tan \theta_{\text {in }}\right| \gg|1-n|$ [so that $\left.\cos \left(\theta_{\text {in }}-\theta_{\text {out }}\right) \approx 1-O(1-n)^{2}\right]$ is the same as

$$
\frac{\cos \theta_{\text {in }}}{\cos \theta_{\text {out }}}=\frac{1}{1+(1-n)-O(1-n)^{2}}=n+O(1-n)^{2},
$$

known as Snell's law.

One should realize that the phase-object approximation only holds if $\left(x_{1}-x_{0}\right)\left(\theta_{\text {in }}-\theta_{\text {out }}\right)^{2} \ll 2 \bar{\lambda}$. This gives an indication of the maximum length of the transition zone between the two media for Eq. (67) or (69) to hold: $x_{1}-x_{0}$ $\ll 2 \bar{\lambda} \tan ^{2} \theta_{i n} /(1-n)^{2}$. Using Eq. (53), this is equal to $x_{1}$ $-x_{0} \ll 8 \tan ^{2} \theta_{i n}\left(2 \pi / k_{c}\right)^{4} / \bar{\lambda}^{3}$. For silicon $\left(k_{c}=0.051 \mathrm{~nm}^{-1}\right)$, $\theta_{i n}=45^{\circ}$, and an average neutron wavelength of $0.2 \mathrm{~nm}, x_{1}$ $-x_{0} \ll 230 \mathrm{~m}$ ! For the region of total reflection, $\theta_{i n} \approx|1-n|$. Then the above condition becomes $x_{1}-x_{0} \ll 2 \bar{\lambda}$, which is not the case and the above derivation is not applicable.

\section{MAGNETIC SCATTERING}

\section{A. Scattering in the sample}

The propagation of the coherence matrix through the sample can be determined by coupling the scattered coherence matrix to the incoming coherence matrix. In general, one can state that

$$
\hat{\Gamma}_{s c}\left(\vec{r}_{1}, \vec{r}_{2}, \tau\right)=\hat{M}_{s c}\left(\vec{r}_{2}\right) \hat{\Gamma}_{i n}\left(\vec{r}_{1}, \vec{r}_{2}, \tau\right) \hat{M}_{s c}\left(\vec{r}_{1}\right)^{\dagger},
$$

where $\hat{M}_{s c}$ is called the scattering matrix. Following the reasoning of Sec. II B, one can split the scattering matrix in a nonmagnetic part and a magnetic part. Using the phaseobject approximation (Sec. IV A), one can write

$$
\hat{M}_{s c}(\vec{r})=e^{i S(\vec{r})} \hat{H}_{m}(\vec{r})
$$

where $\hat{H}_{m}$ is a rotation matrix describing the magnetic part of the scattering matrix. In case of only nonmagnetic scattering, this is just the unit matrix. In the case where the magnetic flux density in the sample is everywhere in the direction of the quantization axis $\vec{e}_{z}$, this is a matrix $\hat{T}_{z}$. Then, the argument of this matrix is the extra phase difference between the up and down spin states acquired by the wave after traveling through the sample. Equation (4) can be used to relate the polarization directly before and after the sample:

$$
\operatorname{Tr}\left(\hat{\Gamma}_{s c}(\vec{r}, \vec{r}, 0)\right)=e^{i S(\vec{r})-i S^{*}(\vec{r})} \operatorname{Tr}\left(\hat{\Gamma}_{i n}(\vec{r}, \vec{r}, 0)\right)
$$

and

$$
\left(\begin{array}{l}
P_{x, s c}(\vec{r}) \\
P_{y, s c}(\vec{r}) \\
P_{z, s c}(\vec{r})
\end{array}\right)=\hat{\Delta}\left(\begin{array}{l}
P_{x, i n}(\vec{r}) \\
P_{y, i n}(\vec{r}) \\
P_{z, i n}(\vec{r})
\end{array}\right)
$$




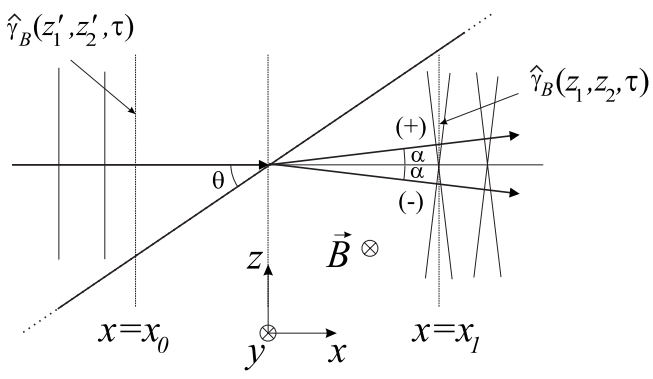

FIG. 5. Polarized beam splitting by a region of constant magnetic flux density.

where $\hat{\Delta}$ is called the depolarization matrix and given by its elements $\Delta_{j k}=\frac{1}{2} \operatorname{Tr}\left(\hat{H}_{m}(\vec{r}) \hat{\sigma}_{k} \hat{H}_{m}(\vec{r})^{\dagger} \hat{\sigma}_{j}\right)$. If the scattering matrix is presented as

$$
\hat{H}_{m}=\hat{I} \cos \theta+i\left(n_{x} \hat{\sigma}_{x}+n_{y} \hat{\sigma}_{y}+n_{z} \hat{\sigma}_{z}\right) \sin \theta,
$$

the depolarization matrix becomes

$$
\begin{aligned}
\hat{\Delta}= & \left(\begin{array}{lll}
1 & 0 & 0 \\
0 & 1 & 0 \\
0 & 0 & 1
\end{array}\right)-2 \sin ^{2} \theta\left(\begin{array}{ccc}
n_{y}^{2}+n_{z}^{2} & -n_{x} n_{y} & -n_{x} n_{z} \\
-n_{y} n_{x} & n_{x}^{2}+n_{z}^{2} & -n_{y} n_{z} \\
-n_{z} n_{x} & -n_{z} n_{y} & n_{x}^{2}+n_{y}^{2}
\end{array}\right) \\
& +2 \sin \theta \cos \theta\left(\begin{array}{ccc}
0 & -n_{z} & n_{y} \\
n_{z} & 0 & -n_{x} \\
-n_{y} & n_{x} & 0
\end{array}\right) .
\end{aligned}
$$

\section{B. Beam splitting}

In the case where the direction of the magnetic flux density in a certain region is parallel to a fixed axis, the propagation of the coherence matrix can be calculated according to the previous section. If a neutron beam propagates along the $x$ axis and the boundary of the magnetic flux density is not perpendicular to the propagation direction, so-called beam splitting is observed, as shown in Fig. 5. The propagation of the coherence matrix is given by Eq. (27). The propagation of the two diagonal elements of this matrix is comparable to the propagation of the mutual coherence function, as given in Sec. IV B. The refraction index is now given by $n^{ \pm}$ $=1 \pm \delta \kappa / \bar{k}$, where + denotes the up spin wave and - the down spin wave. The refraction angle (see Fig. 5) $\alpha$ $=\delta \kappa / \bar{k} \cot \theta$. For a magnetic flux density of $1 / 3 \mathrm{~T}, \theta=45^{\circ}$, and a neutron wavelength of $0.2 \mathrm{~nm}, \alpha \approx 0.5 \mu \mathrm{rad}$. The down spin wave is refracted at the boundary with an angle exactly opposite to the one of the up spin wave. The offdiagonal elements of the coherence matrix propagate in the same way but have an extra phase shift of $\delta \kappa\left(R_{2}+R_{1}\right)$. If $R_{2}+R_{1} \gg \delta \kappa^{-1}$, the value of the integral reduces very fast to 0 . Gähler and co-workers ${ }^{25,26}$ used a space-time view of neutron spin echo in a semiclassical (ray-tracing) picture to explain beam splitting. However, to observe beam splitting, it is needed that Eq. (27) cannot be reduced to Eq. (30). This is the case when the transversal coherence length is of the same order as or larger than $\delta \kappa^{-1}$, as shown in the previous sec- tion. If the transversal coherence length is much smaller than $\delta \kappa^{-1}$, the splitting of the beam can be neglected and Eq. (30) can be used to calculate the propagation of the coherence matrix. Note that the transversal coherence length is also a measure of the divergence of the beam (see Sec. III E). The smaller the beam divergence, the larger the coherence length. Hence, the minimum coherence length required to observe beam splitting can be translated into the maximum allowed beam divergence to observe beam splitting. Clearly, the beam divergence should be less than or of the same order as $\alpha$.

\section{POLARIZATION ANALYSIS}

A typical setup of a neutron polarization manipulating instrument consists of a source, a polarizer and an analyzer, neutron manipulation devices in between, and a detector at the end of the beam. The source can be a reactor or a pulsed source, producing unpolarized neutrons. The polarizer and analyzer are devices which preferentially transmit one spin state over the other. The polarizer creates a polarized beam and the analyzer is used to analyze the polarization of the beam at the exit of the instrument. Here, the device matrix $\hat{D}_{P}$ of a polarizer (polarizing the beam along the quantization direction along the $z$ axis) can be characterized by two functions: the polarizing power $P_{P}$ and the transmission $T_{P}$ :

$$
\hat{D}_{P}\left(\vec{r}^{\prime}, \vec{r}\right)=\sqrt{\frac{T_{P}\left(\vec{r}^{\prime}, \vec{r}\right)}{2}}\left(\begin{array}{cc}
\sqrt{1+P_{P}\left(\vec{r}^{\prime}, \vec{r}\right)} & 0 \\
0 & \sqrt{1-P_{P}\left(\vec{r}^{\prime}, \vec{r}\right)}
\end{array}\right),
$$

and the same for the analyzer with subscript $P$ replaced by $A$. Note that the matrix in this equation is not a rotation matrix except if $P_{P}\left(\vec{r}^{\prime}, \vec{r}\right)=0$. For a directed quasimonochromatic beam, the neutron flux at the detector position is given by

$$
\vec{J}\left(\vec{r}_{d}\right)=2 \vec{v}_{p} \operatorname{Tr}\left(\hat{\Gamma}\left(\vec{r}_{d}, \vec{r}_{d}, 0\right)\right),
$$

which with Eqs. (9) and (30) can be reduced to ( $\cos \theta_{1}$ $\approx \cos \theta_{2} \approx 1$ )

$$
\begin{aligned}
\vec{J}\left(\vec{r}_{d}\right)= & \frac{2 \vec{v}_{p}}{r_{d}^{2} \bar{\lambda}^{2}} \iint_{x=0} e^{-i \vec{p} \cdot\left(\vec{r}_{2}^{\prime}-\vec{r}_{1}^{\prime}\right)} \Gamma_{0}\left(\vec{r}_{1}^{\prime}, \vec{r}_{2}^{\prime}, 0\right) \operatorname{Tr}\left(\hat{M}\left(\vec{r}_{d}, \vec{r}_{2}^{\prime}\right)\right. \\
& \left.\times \hat{\gamma}_{B}\left(\vec{r}_{1}^{\prime}, \vec{r}_{2}^{\prime}, 0\right) \hat{M}\left(\vec{r}_{d}, \vec{r}_{1}^{\prime}\right)^{\dagger}\right) d^{2} r_{1}^{\prime} d^{2} r_{2}^{\prime},
\end{aligned}
$$

where $\vec{p}=\vec{k} \vec{r}_{d} / r_{d}$ and $\hat{M}$ is the matrix describing the whole instrument:

$$
\hat{M}\left(\vec{r}_{d}, \vec{r}^{\prime}\right)=\hat{D}_{A}\left(\vec{r}_{d}, \vec{r}^{\prime}\right) \hat{D}\left(\vec{r}_{d}, \vec{r}^{\prime}\right) \hat{D}_{P}\left(\vec{r}_{d}, \vec{r}^{\prime}\right)
$$

and $\hat{D}$ the overall matrix of the neutron manipulation devices between the analyzer and polarizer, including a possible sample. Remember that the matrix $\hat{M}\left(\vec{r}_{1}, \vec{r}_{0}\right)$ can be calculated as a product of matrices corresponding to regions with a constant magnetic flux density encountered by the neutron traveling in a straight line from $\vec{r}_{0}$ to $\vec{r}_{1}$. If the neutron source is unpolarized, $\hat{\gamma}_{B}\left(\vec{r}_{1}^{\prime}, \vec{r}_{2}^{\prime}, 0\right)=\hat{I} / 2$, yielding 


$$
\begin{aligned}
\vec{J}\left(\vec{r}_{d}\right)= & \frac{\vec{v}_{p}}{r_{d}^{2} \bar{\lambda}^{2}} \iint_{x=0} e^{-i \vec{p} \cdot\left(\vec{r}_{2}^{\prime}-\vec{r}_{1}^{\prime}\right)} \Gamma_{0}\left(\overrightarrow{r_{1}^{\prime}}, \overrightarrow{r_{2}^{\prime}}, 0\right) \operatorname{Tr}\left(\hat{M}\left(\vec{r}_{d}, \overrightarrow{r_{2}^{\prime}}\right)\right. \\
& \left.\times \hat{M}\left(\vec{r}_{d}, \vec{r}_{1}^{\prime}\right)^{\dagger}\right) d^{2} r_{1}^{\prime} d^{2} r_{2}^{\prime} .
\end{aligned}
$$

The neutron flux at the detector position can be calculated from the elements of matrix $\hat{M}$ describing the spin manipulations in the instrument. The matrix describing the action of the analyzer on the neutron spin, $\hat{D}_{A}\left(\vec{r}_{d}, \vec{r}^{\prime}\right)$, will, in general, only depend slightly on $\vec{r}^{\prime}$. Over ranges within the coherence length, it will be constant. Hence,

$$
\hat{D}_{A}\left(\vec{r}_{d}, \vec{r}_{2}^{\prime}\right) \hat{D}_{A}\left(\vec{r}_{d}, \vec{r}_{1}^{\prime}\right)^{\dagger} \approx T_{A}\left(\vec{r}_{1}^{\prime}, \vec{r}_{d}\right) \frac{\hat{I}+P_{A}\left(\vec{r}_{d}, \vec{r}_{1}^{\prime}\right) \hat{\sigma}_{z}}{2}
$$

and the same holds for the polarizer. Using Eq. (79), Eq. (78) can be rewritten as

$$
\begin{aligned}
\vec{J}\left(\vec{r}_{d}\right)= & \frac{2 \vec{v}_{p}}{r_{d}^{2} \bar{\lambda}^{2}} \iint_{x=0} e^{-i \vec{p} \cdot\left(\overrightarrow{r_{2}^{\prime}}-\vec{r}_{1}^{\prime}\right)} \Gamma_{0}\left(\overrightarrow{r_{1}^{\prime}}, \overrightarrow{r_{2}^{\prime}}, 0\right) T_{A}\left(\vec{r}_{1}^{\prime}, \vec{r}_{d}\right) T_{P}\left(\vec{r}_{1}^{\prime}, \vec{r}_{d}\right) \\
& \times \Omega\left(\vec{r}_{d}, \vec{r}_{1}^{\prime}, \vec{r}_{2}^{\prime}\right) d^{2} r_{1}^{\prime} d^{2} r_{2}^{\prime}
\end{aligned}
$$

where

$$
\begin{aligned}
& \Omega\left(\vec{r}_{d}, \vec{r}_{1}^{\prime}, \vec{r}_{2}^{\prime}\right) \\
& \quad=\operatorname{Tr}\left(\frac{\hat{I}+P_{A}\left(\vec{r}_{d}, \vec{r}_{1}^{\prime}\right) \hat{\sigma}_{z}}{2} \hat{D}\left(\vec{r}_{d}, \overrightarrow{r_{2}^{\prime}}\right) \frac{\hat{I}+P_{P}\left(\vec{r}_{d}, \vec{r}_{1}^{\prime}\right) \hat{\sigma}_{z}}{2} \hat{D}\left(\vec{r}_{d}, \vec{r}_{1}^{\prime}\right)^{\dagger}\right) .
\end{aligned}
$$

In general, it is preferable that the results of measurements on samples are as much as possible device independent. However, as is shown in the above equation for the neutron flux at the detector position, many device parameters are still needed. The information on a sample is contained in matrix $\hat{D}$. To reduce the dependences on device parameters like the polarizing powers and transmissions of polarizer and analyzer, one can use so-called spin flippers. It is possible to insert spin flippers just before the analyzer and directly after the polarizer. The flipper influence can be represented by a rotation matrix $\hat{F}\left(\vec{r}_{d}, \vec{r}^{\prime}\right)$ for which holds $\hat{F}\left(\vec{r}_{d}, \vec{r}^{\prime}\right) \hat{F}\left(\vec{r}_{d}, \vec{r}^{\prime}\right)^{\dagger}$ $=1$. Such a matrix will, in general, only depend slightly on $\vec{r}^{\prime}$. Over ranges within the coherence length, it will be constant. Hence, $\hat{F}\left(\vec{r}_{d}, \vec{r}_{2}^{\prime}\right) \hat{F}\left(\vec{r}_{d}, \vec{r}_{1}^{\prime}\right)^{\dagger}=1$. The matrices $\hat{D}$ transform to

$$
\hat{D}^{f}\left(\vec{r}_{d}, \vec{r}^{\prime}\right)=\hat{F}_{A}\left(\vec{r}_{d}, \vec{r}^{\prime}\right) \hat{D}\left(\vec{r}_{d}, \vec{r}^{\prime}\right) \hat{F}_{P}\left(\vec{r}_{d}, \vec{r}^{\prime}\right)
$$

and

$\Omega_{f}\left(\vec{r}_{d}, \vec{r}_{1}^{\prime}, \vec{r}_{2}^{\prime}\right)=\operatorname{Tr}\left(\frac{\hat{I}+P_{A} \hat{F}_{A}^{\dagger} \hat{\sigma}_{z} \hat{F}_{A}}{2} \hat{D}\left(\vec{r}_{d}, \vec{r}_{2}^{\prime}\right) \frac{\hat{I}+P_{P} \hat{F}_{P} \hat{\sigma}_{z} \hat{F}_{P}^{\dagger}}{2} \hat{D}^{\dagger}\right)$,

where the arguments $\left(\vec{r}_{d}, \vec{r}_{1}^{\prime}\right)$ in the trace were omitted for clarity reasons. This enables the definition of the four-shim neutron flux $\vec{J}_{s}^{(4)}\left(\vec{r}_{d}\right)$, the four-flip neutron flux $\vec{J}_{f}^{(4)}\left(\vec{r}_{d}\right)$, and the four-measured polarization $P_{m}^{(4)}\left(\vec{r}_{d}\right)$ :

$$
\begin{aligned}
& J_{s}^{(4)}\left(\vec{r}_{d}\right)=\frac{\left|\vec{J}_{n n}\left(\vec{r}_{d}\right)+\vec{J}_{n f}\left(\vec{r}_{d}\right)+\vec{J}_{f n}\left(\vec{r}_{d}\right)+\vec{J}_{f f}\left(\vec{r}_{d}\right)\right|}{4}, \\
& J_{f}^{(4)}\left(\vec{r}_{d}\right)=\frac{\left|\vec{J}_{n n}\left(\vec{r}_{d}\right)-\vec{J}_{n f}\left(\vec{r}_{d}\right)-\vec{J}_{f n}\left(\vec{r}_{d}\right)+\vec{J}_{f f}\left(\vec{r}_{d}\right)\right|}{4}
\end{aligned}
$$

and

$$
P_{m}^{(4)}\left(\vec{r}_{d}\right)=\frac{J_{f}^{(4)}\left(\vec{r}_{d}\right)}{J_{s}^{(4)}\left(\vec{r}_{d}\right)},
$$

where the indices $f$ and $n$ indicate when the flipper is on (flip) or off (no flip). For perfect flippers, $\hat{F}_{A} \hat{\sigma}_{z} \hat{F}_{A}^{\dagger}=-\hat{\sigma}_{z}$ and the same for $\hat{F}_{P}$ so that $J_{s}^{(4)}\left(\vec{r}_{d}\right)$ is given by Eq. (82) with $\Omega$ replaced by

$$
\Omega_{s}^{(4)}\left(\vec{r}_{d}, \vec{r}_{1}^{\prime}, \vec{r}_{2}^{\prime}\right)=\frac{1}{4} \operatorname{Tr}\left(\hat{D}\left(\vec{r}_{d}, \vec{r}_{2}^{\prime}\right) \hat{D}\left(\vec{r}_{d}, \vec{r}_{1}^{\prime}\right)^{\dagger}\right)
$$

independent of the polarizer or analyzer properties. Under the same conditions, $J_{f}^{(4)}\left(\vec{r}_{d}\right)$ becomes

$$
\begin{aligned}
J_{f}^{(4)}\left(\vec{r}_{d}\right)= & \iint_{x=0} e^{-i \vec{p} \cdot\left(\overrightarrow{r_{2}^{\prime}}-\vec{r}_{1}^{\prime}\right)} \Gamma_{0}\left(\vec{r}_{1}^{\prime}, \vec{r}_{2}^{\prime}, 0\right) T_{A}\left(\vec{r}_{d}, \vec{r}_{1}^{\prime}\right) T_{P}\left(\vec{r}_{d}, \vec{r}_{1}^{\prime}\right) \\
& \times \Omega_{m}^{(4)}\left(\vec{r}_{d}, \overrightarrow{r_{1}^{\prime}}, \vec{r}_{2}^{\prime}\right) d^{2} r_{1}^{\prime} d^{2} r_{2}^{\prime},
\end{aligned}
$$

where

$$
\begin{aligned}
& \Omega_{m}^{(4)}\left(\vec{r}_{d}, \vec{r}_{1}^{\prime}, \vec{r}_{2}^{\prime}\right) \\
& \quad=\frac{1}{4} P_{A}\left(\vec{r}_{d}, \vec{r}_{1}^{\prime}\right) P_{P}\left(\vec{r}_{d}, \vec{r}_{1}^{\prime}\right) \operatorname{Tr}\left(\hat{\sigma}_{z} \hat{D}\left(\vec{r}_{d}, \vec{r}_{2}^{\prime}\right) \hat{\sigma}_{z} \hat{D}\left(\vec{r}_{d}, \vec{r}_{1}^{\prime}\right)^{\dagger}\right) .
\end{aligned}
$$

Most of the time, instead of using four quantities, the shim and polarization are determined by two quantities. The twoshim neutron flux, two-flip neutron flux and two-measured polarization are defined as

$$
\begin{aligned}
& J_{s}^{(2)}\left(\vec{r}_{d}\right)=\frac{\left|\vec{J}_{n}\left(\vec{r}_{d}\right)+\vec{J}_{f}\left(\vec{r}_{d}\right)\right|}{2}, \\
& J_{f}^{(2)}\left(\vec{r}_{d}\right)=\frac{\left|\vec{J}_{n}\left(\vec{r}_{d}\right)-\vec{J}_{f}\left(\vec{r}_{d}\right)\right|}{2},
\end{aligned}
$$

and

$$
P_{m}^{(2)}\left(\vec{r}_{d}\right)=\frac{J_{f}^{(2)}\left(\vec{r}_{d}\right)}{J_{s}^{(2)}\left(\vec{r}_{d}\right)},
$$

where the indices $f$ or $n$ indicates when a flipper is on or off. The flipper can be either the one just before the analyzer or the one after the polarizer. If the flipper at the analyzer is used and assumed perfect, $\vec{J}_{s}^{(2)}\left(\vec{r}_{d}\right)$ is given by Eq. (82) with $\Omega$ replaced by 


$$
\Omega_{s}^{(2)}\left(\vec{r}_{d}, \vec{r}_{1}^{\prime}, \vec{r}_{2}^{\prime}\right)=\frac{1}{2} \operatorname{Tr}\left(\hat{D}\left(\vec{r}_{d}, \vec{r}_{1}^{\prime}\right)^{\dagger} \hat{D}\left(\vec{r}_{d}, \vec{r}_{2}^{\prime}\right) \frac{\hat{I}+P_{P}\left(\vec{r}_{d}, \vec{r}_{1}^{\prime}\right) \hat{\sigma}_{z}}{2}\right) .
$$

Under the same conditions, $J_{f}^{(2)}\left(\vec{r}_{d}\right)$ is given by Eq. (90) with $\Omega_{m}^{(4)}\left(\vec{r}_{d}, \vec{r}_{1}^{\prime}, \vec{r}_{2}^{\prime}\right)$ replaced by

$$
\begin{aligned}
& \Omega_{m}^{(2)}\left(\vec{r}_{d}, \vec{r}_{1}^{\prime}, \vec{r}_{2}^{\prime}\right) \\
& \quad=\frac{P_{A}\left(\vec{r}_{d}, \vec{r}_{1}^{\prime}\right)}{2} \operatorname{Tr}\left(\hat{D}\left(\vec{r}_{d}, \vec{r}_{1}^{\prime}\right)^{\dagger} \hat{\sigma}_{z} \hat{D}\left(\vec{r}_{d}, \vec{r}_{2}^{\prime}\right) \frac{\hat{I}+P_{P}\left(\vec{r}_{d}, \vec{r}_{1}^{\prime}\right) \hat{\sigma}_{z}}{2}\right) .
\end{aligned}
$$

Equations when the flipper is at the polarizer side can be derived in a similar manner. Note that, in general, the differ-

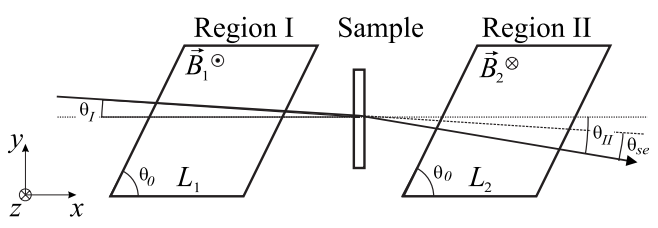

FIG. 6. Principle of SESANS angle coding.

ent definitions of shim neutron flux and measured polarization give different results. In a special case, however, they are the same. If matrix $\hat{D}$ only slightly depends on $\vec{r}^{\prime}$, then within the coherence length, it will be constant: $\hat{D}\left(\vec{r}_{d}, \vec{r}_{2}^{\prime}\right)$ $\approx \hat{D}\left(\vec{r}_{d}, \vec{r}_{1}^{\prime}\right)$. If also $\hat{D}\left(\vec{r}_{d}, \vec{r}^{\prime}\right)$ can be represented by a rotation matrix:

$$
\hat{D}\left(\vec{r}_{d}, \vec{r}^{\prime}\right)=\frac{1}{\sqrt{2}}\left(\begin{array}{cc}
\sqrt{1+P_{D}\left(\vec{r}_{d}, \vec{r}^{\prime}\right)} e^{i \alpha_{D}\left(\vec{r}_{d}, \vec{r}^{\prime}\right)} & i \sqrt{1-P_{D}\left(\vec{r}_{d}, \vec{r}^{\prime}\right)} e^{i \beta_{D}\left(\vec{r}_{d}, \vec{r}^{\prime}\right)} \\
i \sqrt{1-P_{D}\left(\vec{r}_{d}, \vec{r}^{\prime}\right)} e^{-i \beta_{D}\left(\vec{r}_{d}, \vec{r}^{\prime}\right)} & \sqrt{1+P_{D}\left(\vec{r}_{d}, \vec{r}^{\prime}\right)} e^{-i \alpha_{D}\left(\vec{r}_{d}, \vec{r}^{\prime}\right)}
\end{array}\right),
$$

then $\Omega_{s}^{(4)}\left(\vec{r}_{d}, \vec{r}_{1}^{\prime}, \vec{r}_{2}^{\prime}\right)=\Omega_{s}^{(2)}\left(\vec{r}_{d}, \vec{r}_{1}^{\prime}, \vec{r}_{2}^{\prime}\right)=\frac{1}{2}$ and the shim neutron flux is independent of all neutron manipulation devices. Also, $\Omega_{m}^{(4)}$ and $\Omega_{m}^{(2)}$ are reduced to

$$
\begin{aligned}
\Omega_{m}^{(4)}\left(\vec{r}_{d}, \vec{r}_{1}^{\prime}, \vec{r}_{2}^{\prime}\right) & =\Omega_{m}^{(2)}\left(\vec{r}_{d}, \vec{r}_{1}^{\prime}, \vec{r}_{2}^{\prime}\right) \\
& =\frac{1}{2} P_{A}\left(\vec{r}_{d}, \vec{r}_{1}^{\prime}\right) P_{P}\left(\vec{r}_{d}, \vec{r}_{1}^{\prime}\right) P_{D}\left(\vec{r}_{d}, \vec{r}_{1}^{\prime}\right),
\end{aligned}
$$

describing the loss of polarization due to the neutron manipulating devices. Hence, the two-shim neutron flux and the two-measured polarization can be used if the device matrix between the polarizer and analyzer is a rotation matrix which does not change within the coherence length of the neutron beam.

\section{SPIN ECHO SMALL ANGLE NEUTRON SCATTERING}

\section{A. Introduction}

A neutron spin echo instrument consists of two regions where the neutron spin performs precessions depending on some property of the neutron and its path through these regions. For conventional spin echo, the important property that is coded is the neutron wavelength ${ }^{27}$ enabling inelastic measurements with a high time resolution. It is also possible to code the angle a neutron has taken through the precession regions. Assuming that no inelastic scattering occurs, the total precession is proportional to the difference in angles of the path through the first region and the second region. This is called SESANS. ${ }^{18,20}$ The basis for this technique was first discussed by Mezei in 1972 (Ref. 27) and Pynn in 1978. ${ }^{28}$ Keller et al. ${ }^{29}$ showed in 1995 that it could be used for small angle neutron scattering and Rekveldt ${ }^{18}$ (in 1996) was the first to consider the mathematical background of the technique and introduced the SESANS correlation function, as will be discussed later.

The principle of the coding part of a SESANS setup is shown in Fig. 6. Regions I and II are parallelogram shaped regions with lengths $L_{1}$ and $L_{2}$. The dependence in the $z$ direction is ignored. In region $\mathrm{I}$, the magnetic flux density $\left(B_{1}\right)$ is constant and parallel to the $z$ axis. In region II, the magnetic flux density $\left(B_{2}\right)$ is the same but in the opposite direction. Outside these regions, the magnetic flux density is assumed to be 0 . The inclination angle between region I and the neutron beam is $\theta_{0}$. After interaction with the sample, the angle the neutron path makes with region II is changed by $\theta_{s e}$. The influences on the propagation of the coherence matrix in regions I and II are given by Eq. (21) where terms of second and larger orders in $\theta_{\mathrm{I}}$ and $\theta_{\mathrm{I}}$ are ignored:

$$
\phi_{\mathrm{I}}\left(\overrightarrow{r^{\prime}}, \vec{r}\right)=-c \vec{\lambda} B_{1} L_{1}\left(1-\theta_{\mathrm{I}} / \tan \theta_{0}\right)
$$

and

$$
\phi_{\mathrm{II}}\left(\overrightarrow{r^{\prime}}, \vec{r}\right)=c \bar{\lambda} B_{2} L_{2}\left(1-\theta_{\mathrm{II}} / \tan \theta_{0}\right),
$$

where $c=4.63209 \times 10^{14} \mathrm{~T}^{-1} \mathrm{~m}^{-2}$, as defined in Sec. II D. Hence, the device matrix for the regions I and II is given by $\hat{T}_{z}\left(\phi_{\mathrm{I}}\left(\vec{r}^{\prime}, \vec{r}\right)\right)$ and $\hat{T}_{z}\left(\phi_{\mathrm{II}}\left(\vec{r}^{\prime}, \vec{r}\right)\right)$. If the sample scatters nonmagnetically and $B_{1} L_{1}=B_{2} L_{2}$, the device matrix for region I, the sample, and region II is just $\hat{T}_{z}\left(\vec{l}_{s e} \cdot \vec{q}_{s e}\right)$, where $\vec{q}_{s e}$ is the wave vector transfer at the sample position, the direction of $\vec{l}_{s e}$ is the coding direction (here $\vec{e}_{y}$ ), and $l_{s e}=c \bar{\lambda}^{2} B L /$ $2 \pi \tan \theta_{0}$ is called the spin echo length. For a complete SESANS instrument, the coding section is part of the whole instrument. This is shown schematically in Fig. 7. The neu- 


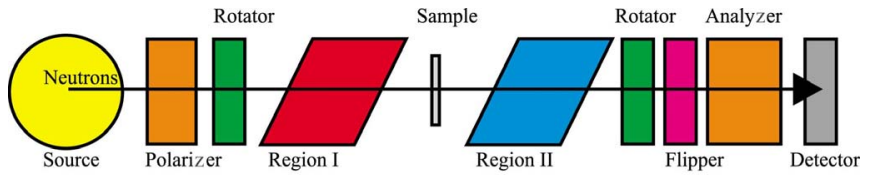

$y_{\Delta}$<smiles>[Z][Co][CH]</smiles>

FIG. 7. (Color online) Principle of complete SESANS setup.

trons from the source are transmitted through the polarizer and the rotator rotates the polarization vector to the $y$ axis. This corresponds to a device matrix which consist of a rotation matrix, $\hat{R}\left(\vec{r}^{\prime}, \vec{r}\right)$. Then, the neutron propagates through regions I and II and a second rotator which reverses the previous rotator, so its device matrix is the inverse (or Hermitian adjunct) of the previous one, $\hat{R}\left(\vec{r}^{\prime}, \vec{r}\right)^{\dagger}$. Then, the neutrons move through a flipper and analyzer to enable polarization analysis. Now, the device matrix between the polarizer and flipper directly in front of the analyzer is given by

$$
\hat{E}\left(\vec{r}, \overrightarrow{r^{\prime}}\right)=\hat{R}\left(\vec{r}^{\prime}, \vec{r}\right)^{\dagger} \hat{T}_{z}\left(\phi_{\mathrm{II}}\left(\overrightarrow{r^{\prime}}, \vec{r}\right)\right) \hat{M}_{s c}\left(\vec{r}, \overrightarrow{r^{\prime}}\right) \hat{T}_{z}\left(\phi_{\mathrm{I}}\left(\overrightarrow{r^{\prime}}, \vec{r}\right)\right) \hat{R}\left(\overrightarrow{r^{\prime}}, \vec{r}\right) .
$$

Note that without a scattering sample, this matrix reduces to the identity matrix. If the scattering is nonmagnetic, this matrix reduces to

$$
\hat{E}\left(\vec{r}, \vec{r}^{\prime}\right)=e^{i S(\vec{r})} \hat{R}\left(\vec{r}^{\prime}, \vec{r}\right)^{\dagger} \hat{T}_{z}\left(\vec{l}_{s e} \cdot \vec{q}_{s e}\right) \hat{R}\left(\vec{r}^{\prime}, \vec{r}\right)
$$

The polarizing factor of $\hat{E}\left(\vec{r}, \vec{r}^{\prime}\right)$ is

$$
P_{E}\left(\vec{r}, \vec{r}^{\prime}\right)=\left[1-P_{R}^{2}\left(\vec{r}^{\prime}, \vec{r}\right)\right] \cos \left\{\vec{l}_{s e} \cdot \vec{q}_{s e}\right\}+P_{R}^{2}\left(\vec{r}^{\prime}, \vec{r}\right),
$$

where $P_{R}$ is the polarizing factor of $\hat{R}\left(\vec{r}^{\prime}, \vec{r}\right)$. For a perfect spin echo instrument, the factor before the cosine must be maximal and the other term minimal; hence, $P_{R}=0$.

\section{B. Nonmagnetic scattering}

Assume that the neutron beam is homogeneous and unpolarized before the polarizer; then, it is represented by a coherence matrix $\hat{\Gamma}_{0}\left(\vec{r}_{1}^{\prime \prime}, \vec{r}_{2}^{\prime \prime}, \tau\right)=\Gamma_{0}\left(0, \vec{r}^{\prime \prime}, \tau\right) \hat{I} / 2$, where $\Gamma_{0}\left(0, \vec{r}^{\prime \prime}, \tau\right)$ is the mutual coherence function at the source position. The difference in distance between two points in the source plane and two points at the sample plane is $R_{2}$ $-R_{1} \approx\left(\vec{r}^{\prime \prime}-\vec{r}^{\prime}\right) \cdot\left(\vec{s}_{0}-\vec{s}_{1}\right) / r_{s}$, where $\vec{s}_{0}$ is the component in the $(y, z)$ plane at the source position of the first point at the source and $\vec{s}_{0}+\vec{r}$ "the same of the second point at the source, $\vec{s}_{1}$ is the component in the $(y, z)$ plane at the sample position of the first point at the sample and $\vec{s}_{1}+\vec{r}^{\prime}$ the same for the second point at the sample, and $r_{s}$ is the distance along the $x$ axis between the source and sample. The approximation holds as long as $\bar{k}\left|\vec{r}^{\prime \prime}-\vec{r}^{\prime}\right|^{2} \ll r_{s}$. Using Eq. (30) and $\cos \theta_{1}$ $\approx \cos \theta_{2} \approx 1$, the coherence matrix at the sample position becomes

$$
\begin{aligned}
& \hat{\Gamma}_{i n}\left(\vec{s}_{1}, \vec{s}_{1}+\vec{r}^{\prime}, \tau\right) \\
& \quad=\frac{1}{2 r_{s}^{2}} \int_{A_{0}} R_{i n}\left(\vec{q}_{0}-\vec{q}_{1}, \vec{r}^{\prime}, \tau\right) \hat{W}_{P}\left(\vec{r}_{1}^{\prime}, \vec{r}_{1}^{\prime \prime}\right) \hat{W}_{P}\left(\vec{r}_{1}^{\prime}, \vec{r}_{1}^{\prime \prime}\right)^{\dagger} d^{2} s_{0}
\end{aligned}
$$

and

$$
R_{i n}\left(\vec{q}, \overrightarrow{r^{\prime}}, \tau\right)=\frac{e^{-i \vec{q} \cdot \overrightarrow{r^{\prime}}}}{\bar{\lambda}^{2}} \int_{A_{0}} e^{i \vec{q} \cdot \overrightarrow{r^{\prime \prime}}} \Gamma_{0}\left(0, \overrightarrow{r^{\prime \prime}}, \tau\right) d^{2} r^{\prime \prime}
$$

where $\quad \vec{q}_{0}=\bar{k}_{s_{0}} / r_{s}, \quad \vec{q}_{1}=\vec{k}_{s_{1}} / r_{s}, \quad$ and $\quad \hat{W}_{P}\left(\vec{r}_{1}, \vec{r}_{2}\right)$ $=\hat{M}_{P}\left(\vec{r}_{1}, \vec{r}_{2}\right) \hat{D}_{P}\left(\vec{r}_{1}, \vec{r}_{2}\right) . \hat{D}_{P}\left(\vec{r}_{1}, \vec{r}_{2}\right)$ is the device matrix of the polarizer and $\hat{M}_{P}\left(\vec{r}_{1}, \vec{r}_{2}\right)$ represents the device matrix of the parts between the polarizer and sample position. For a complete incoherent source (see Sec. III B):

$$
R_{i n}\left(\vec{q}, \overrightarrow{r^{\prime}}, \tau\right)=\frac{J_{0} e^{-i \bar{k} v_{p} \tau} e^{-i \vec{q} \cdot \overrightarrow{r^{\prime}}}}{8 v_{p} \pi}
$$

The propagation from just behind the sample to the detector is detailed as follows. The difference in distance between two points in the sample plane and one point at the detector plane is $R_{2}-R_{1} \approx \vec{r}^{\prime} \cdot\left(\vec{s}_{1}-\vec{s}_{d}\right) / r_{d}$, where $\vec{s}_{d}$ is the component in the $(y, z)$ plane at the detector position of the point at the detector and $r_{d}$ the distance along the $x$ axis between the sample and detector. The approximation holds as long as $\bar{k}\left|\vec{r}^{\prime}\right|^{2} \ll r_{d}$. Using Eq. (30) and $\cos \theta_{1} \approx \cos \theta_{2} \approx 1$, the coherence matrix at the detector position becomes

$$
\begin{aligned}
\hat{\Gamma}_{d}\left(\vec{r}_{d}, \vec{r}_{d}, \tau\right)= & \frac{1}{r_{d}^{2} \bar{\lambda}^{2}} \int_{A_{s}} \int_{A_{s}} e^{i\left[\left(r_{s} / r_{d}\right) \vec{q}_{1}-\vec{q}_{d}\right] \cdot \vec{r}^{\prime}} \hat{W}_{A}\left(\vec{r}_{d}, \vec{r}_{1}^{\prime}\right) \\
& \times \hat{\Gamma}_{s c}\left(\vec{s}_{1}, \vec{s}_{1}+\vec{r}^{\prime}, \tau\right) \hat{W}_{A}\left(\vec{r}_{d}, \vec{r}_{1}^{\prime}\right)^{\dagger} d^{2} s_{1} d^{2} r^{\prime},
\end{aligned}
$$

where $\hat{\Gamma}_{s c}\left(\vec{s}_{1}, \vec{s}_{1}+\vec{r}^{\prime}, \tau\right)$ is the coherence matrix just behind scattering in the sample and $\vec{q}_{d}=\bar{k} \vec{s}_{d} / r_{d} . \hat{W}_{A}\left(\vec{r}_{d}, \vec{r}_{1}^{\prime}\right)$ $=\hat{D}_{A}\left(\vec{r}_{d}, \vec{r}_{1}^{\prime}\right) \hat{M}_{A}\left(\vec{r}_{d}, \vec{r}_{1}^{\prime}\right)$, where $\hat{D}_{A}\left(\vec{r}_{d}, \vec{r}_{1}^{\prime}\right)$ is the device matrix of the analyzer and $\hat{M}_{A}\left(\vec{r}_{d}, \vec{r}_{1}^{\prime}\right)$ represents the device matrix of the parts between the sample position and analyzer. For nonmagnetic scattering, the scattering matrix [see Eq. (71)] reduces to the identity matrix times a phase factor and the scattered coherence matrix is

$$
\hat{\Gamma}_{s c}\left(\vec{s}_{1}, \vec{s}_{1}+\vec{r}^{\prime}, \tau\right)=e^{i S\left(\vec{s}_{1}+\vec{r}^{\prime}\right)-i S^{*}\left(\vec{s}_{1}\right)} \hat{\Gamma}_{i n}\left(\vec{s}_{1}, \vec{s}_{1}+\vec{r}^{\prime}, \tau\right)
$$

As in Sec. III C, the detector count rate is determined as an integral of the neutron flux over the detector area. Using Eq. (6), this reduces to

$$
I_{d}=2 v_{p} \int_{A_{d}} \operatorname{Tr}\left(\hat{\Gamma}\left(\vec{r}_{d}, \vec{r}_{d}, 0\right)\right) d^{2} r_{d} .
$$

After changing the integration order, the neutron count rate in the detector according to this equation becomes 


$$
\begin{aligned}
I_{d}= & \frac{v_{p}}{r_{s}^{2} r_{d}^{2}-\int^{2}} \int_{A_{0}} \int_{A_{s}} \int_{A_{s}} e^{i\left(r_{s} / r_{d}\right) \vec{q}_{1} \cdot \vec{r}^{\prime}} e^{i S\left(\vec{s}_{1}+\vec{r}^{\prime}\right)-i S^{*}\left(\vec{s}_{1}\right)} \\
& \times R_{i n}\left(\vec{q}_{0}-\vec{q}_{1}, \overrightarrow{r^{\prime}}, 0\right) X\left(\overrightarrow{r^{\prime}}, \overrightarrow{r_{1}^{\prime}}, \overrightarrow{r_{1}^{\prime \prime}}\right) d^{2} r^{\prime} d^{2} s_{1} d^{2} s_{0},
\end{aligned}
$$

where

$$
X\left(\overrightarrow{r^{\prime}}, \overrightarrow{r_{1}^{\prime}}, \overrightarrow{r_{1}^{\prime \prime}}\right)=\int_{A_{d}} e^{-i \vec{q}_{d}} \overrightarrow{r^{\prime}} \Omega_{d}\left(\overrightarrow{r_{d}}, \overrightarrow{r_{1}^{\prime}}, \overrightarrow{r_{1}^{\prime \prime}}\right) d^{2} r_{d}
$$

and

$$
\begin{aligned}
& \Omega_{d}\left(\vec{r}_{d}, \overrightarrow{r_{1}^{\prime}}, \overrightarrow{r_{1}^{\prime \prime}}\right)=T_{A}\left(\vec{r}_{d}, \vec{r}_{1}^{\prime}\right) T_{P}\left(\overrightarrow{r_{1}^{\prime}}, \overrightarrow{r_{1}^{\prime \prime}}\right) \operatorname{Tr}\left(\frac{\hat{I}+P_{A}\left(\vec{r}_{d}, \vec{r}_{1}^{\prime}\right) \sigma_{z}}{2}\right. \\
& \left.\quad \times \hat{E}\left(\vec{r}_{d}, \vec{r}_{1}^{\prime}, \overrightarrow{r_{1}^{\prime \prime}}\right) \frac{\hat{I}+P_{P}\left(\overrightarrow{r_{1}^{\prime}}, \overrightarrow{r_{1}^{\prime \prime}}\right) \sigma_{z}}{2} \hat{E}\left(\vec{r}_{d}, \overrightarrow{r_{1}^{\prime}}, \overrightarrow{r_{1}^{\prime \prime}}\right)^{\dagger}\right)
\end{aligned}
$$

where $\hat{E}\left(\vec{r}_{d}, \vec{r}_{1}^{\prime}, \vec{r}_{1}^{\prime \prime}\right)=\hat{M}_{A}\left(\vec{r}_{d}, \vec{r}_{1}^{\prime}\right) \hat{M}_{P}\left(\vec{r}_{1}^{\prime}, \vec{r}_{1}^{\prime \prime}\right)$. In the following, the transmission of the analyzer and polarizer is assumed to be constant. Again, to make the measurement results independent of the device parameters, measurements are performed of the two-shim count rate and the two-measured polarization. The shim count rate can be calculated by taking $\Omega_{d}=\frac{1}{2} T_{A} T_{P}$ so that $X$ can be replaced by

$$
X_{s}\left(\overrightarrow{r^{\prime}}, \overrightarrow{r_{1}^{\prime}}, \overrightarrow{r_{1}^{\prime \prime}}\right)=\frac{T_{A} T_{P}}{2} \frac{r_{d}^{2}}{\bar{k}^{2}} \int_{A_{d}} e^{-i \vec{q}_{d} \cdot \vec{r}^{\prime}} d^{2} q_{d},
$$

where the integral of $s_{d}$ over $A_{d}$ is replaced by an integral of $\vec{q}_{d}$. Now, if the detector area is large enough, the integral over $A_{d}$ is just a two-dimensional Dirac delta function $4 \pi^{2} \delta^{(2)}\left(\vec{r}^{\prime}\right)$. This reduces the shim count rate to

$$
I_{s}=\frac{v_{p}}{r_{s}^{2}} \frac{T_{A} T_{P}}{2} \int_{A_{0}} \int_{A_{s}} e^{i S\left(\vec{s}_{1}\right)-i S^{*}\left(\vec{s}_{1}\right)} R_{i n}\left(\vec{q}_{0}-\vec{q}_{1}, 0,0\right) d^{2} s_{1} d^{2} s_{0},
$$

which, for a complete incoherent source, can be reduced to

$$
I_{s}=\frac{T_{A} T_{P}}{4} \frac{J_{0} A_{0}}{4 \pi r_{s}^{2}} G_{r}(0)
$$

where

$$
G_{r}(0)=\int_{A_{s}} e^{i S\left(\vec{s}_{1}\right)-i S^{*}\left(\vec{s}_{1}\right)} d^{2} s_{1}
$$

is the sample correlation function for $\vec{r}=0$ as defined in Eq. (59). If also the polarization powers of the analyzer and polarizer are constant and the matrix $\hat{E}$ only depends on the angle of the paths before and after the sample, the flip count rate can be calculated by taking $\Omega_{d}$ equal to

$$
\Omega_{f}\left(\vec{q}_{s e}\right)=\frac{1}{2} T_{A} T_{P} P_{A} P_{P} P_{E}\left(\vec{q}_{s e}\right)
$$

where $P_{E}\left(\vec{q}_{s e}\right)$ is the polarizing factor of the setup between the flippers as given by Eq. (103) for a perfect spin echo instrument $P_{E}\left(\vec{q}_{s e}\right)=\cos \left\{\vec{l}_{s e} \cdot \vec{q}_{s e}\right\}$ and $\vec{q}_{s e}=\vec{q}_{d}+\vec{q}_{0}-\vec{q}_{1}\left(r_{s} / r_{d}\right.$ $+1)$. Now, the flip count rate can be found by replacing $X$ by

$$
\begin{aligned}
X_{f}\left(\overrightarrow{r^{\prime}}, \overrightarrow{r_{1}^{\prime}}, \overrightarrow{r_{1}^{\prime \prime}}\right)= & \frac{T_{A} T_{P} P_{A} P_{P} r_{d}^{2}}{2 \vec{k}^{2}} \\
& \times \Re\left\{e^{i \vec{l}_{s e} \cdot\left[\vec{q}_{0}-\vec{q}_{1}\left(r_{s} / r_{d}+1\right)\right]} \int_{A_{d}} e^{-i \vec{q}_{d} \cdot\left(\overrightarrow{r^{\prime}}-\vec{l}_{s e}\right)} d^{2} q_{d}\right\},
\end{aligned}
$$

where the integral of $s_{d}$ over $A_{d}$ is replaced by an integral of $\vec{q}_{d}$. Now again, if the detector area is large enough, the integral over $A_{d}$ is just a two-dimensional Dirac delta function $4 \pi^{2} \delta^{(2)}\left(\vec{r}^{\prime}-\vec{l}_{s e}\right)$. This reduces the flip count rate for a complete incoherent source to

$$
\frac{I_{f}}{I_{0}}=\Re\left\{G_{r}\left(\vec{l}_{s e}\right)\right\},
$$

where $I_{0}=P_{A} P_{P} \frac{T_{A} T_{P}}{4} \frac{J_{0} A_{0}}{4 \pi r_{s}^{2}}$. The measured polarization becomes

$$
\frac{P_{m}}{P_{A} P_{P}}=\frac{\Re\left\{G_{r}\left(\vec{l}_{s e}\right)\right\}}{G_{r}(0)}
$$

so that the measured polarization is proportional to the real part of the SESANS correlation function. The correlation is measured in the coding direction $\vec{l}_{s e}$. One should realize that the above equation only holds for scattering in the phaseobject approximation. A further limitation is that it holds for an ideal spin echo instrument, where the polarizing powers and transmissions of the polarizer and analyzer are constant and the polarizing factor of the setup between the polarizer and analyzer is proportional to $\cos \left\{\vec{l}_{s e} \cdot \vec{q}_{s e}\right\}$. If this is not the case, one can try to solve Eq. (110) differently or by means of numerical calculations.

\section{Magnetic scattering}

Recently, Grigoriev et al., ${ }^{30,31}$ have shown the possibility of spin echo small angle neutron scattering measurements for magnetic samples. For magnetic scattering, the scattering matrix should be incorporated. Here, it is assumed that the source is completely incoherent and the polarizing powers and transmissions of the polarizer and analyzer are constant. Further, the rotation matrices before and after the precession regions I and II are ideal and given by

$$
\hat{R}=\frac{1}{\sqrt{2}}\left(\begin{array}{cc}
e^{i \alpha} & i e^{i \beta} \\
i e^{-i \beta} & e^{-i \alpha}
\end{array}\right),
$$

so that $\hat{R} \sigma_{z} \hat{R}^{\dagger}=T_{z}(2 \alpha+2 \beta) \sigma_{y}$. The detector count rate can be calculated according to Eq. (110) where $\Omega_{d}$ is replaced by

$$
\begin{aligned}
\Omega_{d}\left(\vec{r}_{d}, \overrightarrow{r_{1}^{\prime}}, \overrightarrow{r^{\prime}}, \overrightarrow{r_{1}^{\prime \prime}}\right)= & \frac{T_{A} T_{P}}{4} \operatorname{Tr}\left(\hat{H}_{2} \hat{H}_{1}+P_{A} \hat{\sigma}_{y} \hat{T}_{z}\left(\phi_{2}\right) \hat{H}_{2} \hat{H}_{1} \hat{T}_{z}\left(\phi_{2}\right)^{\dagger}\right. \\
& +P_{P} \hat{\sigma}_{y} \hat{T}_{z}\left(\phi_{1}\right)^{\dagger} \hat{H}_{1} \hat{H}_{2} \hat{T}_{z}\left(\phi_{1}\right)+P_{P} P_{A} \hat{T}_{z}( \\
& \left.\left.-2 \phi_{2}\right) \hat{\sigma}_{y} \hat{H}_{2} \hat{T}_{z}\left(2 \phi_{1}\right) \hat{\sigma}_{y} \hat{H}_{1}\right),
\end{aligned}
$$

where $\phi_{2}=\phi_{\mathrm{II}}-\alpha-\beta$ and $\phi_{1}=\phi_{\mathrm{I}}+\alpha+\beta$ and $\hat{H}_{1}=\hat{H}_{m}\left(\vec{s}_{1}\right)^{\dagger}$ and $\hat{H}_{2}=\hat{H}_{m}\left(\vec{s}_{1}+\vec{r}^{\prime}\right)$. Note that the second and third terms contain 
either $\phi_{1}$ or $\phi_{2}$. As $\phi_{1}$ and $\phi_{2}$ vary fast with the position and angle, these terms will not contribute to the detector count rate. The integrals in Eq. (110) of these terms yield 0. This also collapses the difference between the two-shim neutron flux and four-shim neutron flux and the same for the flip neutron fluxes and measured polarizations. If the values of $\phi_{1}$ or $\phi_{2}$ are large enough, the shim count rate is represented by the first term and the flip count rate by the fourth term. Filling in Eq. (110) for the shim count rate and assuming the detector area is large enough gives

$$
I_{s}=\frac{v_{p}}{r_{s}^{2}} \frac{T_{A} T_{P}}{2} \int_{A_{0}} \int_{A_{s}} e^{i S\left(\vec{s}_{1}\right)-i S^{*}\left(\vec{s}_{1}\right)} R_{i n}\left(\vec{q}_{0}-\vec{q}_{1}, 0,0\right) d^{2} s_{1} d^{2} s_{0},
$$

not dependent on the magnetic scattering, the same result as for a nonmagnetic sample. To calculate the flip count rate, remember that any rotation matrix can be written as a matrix sum of a matrix representing a rotation along the $z$ axis and a flipping matrix. In this case, spin dependent reflection and absorption phenomena are ignored as follows from the phase-object approximation. If these phenomena are to be taken into account, the following derivation should be adjusted. For now, the scattering matrix $\hat{H}_{m}$ can be written as a sum of $\hat{T}_{z}$ and $\hat{F}_{z}$ :

$$
\hat{H}_{m}=\sqrt{\frac{1+P_{m}}{2}} \hat{T}_{z}\left(2 \gamma_{m}\right)+\sqrt{\frac{1-P_{m}}{2}} \hat{F}_{z}\left(2 \delta_{m}\right)
$$

so that

$$
\begin{aligned}
\operatorname{Tr}\left(\hat{T}_{z}(\right. & \left.\left.-2 \phi_{2}\right) \hat{\sigma}_{y} \hat{H}_{m}\left(\vec{s}_{1}+\vec{r}^{\prime}\right) \hat{T}_{z}\left(2 \phi_{1}\right) \hat{\sigma}_{y} \hat{H}_{m}\left(\vec{s}_{1}\right)^{\dagger}\right) \\
= & \sqrt{1+P_{m}\left(\vec{s}_{1}+\vec{r}^{\prime}\right)} \sqrt{1+P_{m}\left(\vec{s}_{1}\right)} \cos \left(\phi_{1}+\phi_{2}+\gamma_{m}\left(\vec{s}_{1}+\vec{r}^{\prime}\right)\right. \\
& \left.+\gamma_{m}\left(\vec{s}_{1}\right)\right)-\sqrt{1-P_{m}\left(\vec{s}_{1}+\vec{r}^{\prime}\right)} \sqrt{1-P_{m}\left(\vec{s}_{1}\right)} \\
& \times \cos \left(\phi_{2}-\phi_{1}+\delta_{m}\left(\vec{s}_{1}\right)+\delta_{m}\left(\vec{s}_{1}+\vec{r}^{\prime}\right)\right),
\end{aligned}
$$

The second term of Eq. (125) depends on the difference $\phi_{2}$ $-\phi_{1}$, which, according to Eqs. (99) and (100) also varies fast with angle and position. Hence, this term also averages to 0 . Hence, again by filling in Eq. (110), the flip count rate is given by

$$
\begin{aligned}
I_{f}= & \frac{v_{p}}{r_{s}^{2} r_{d}^{2} \bar{\lambda}^{2}} \int_{A_{0}} \int_{A_{s}} \int_{A_{s}} e^{i\left(r_{s} / r_{d}\right) \overrightarrow{q_{1}} \cdot \overrightarrow{r^{\prime}}} e^{i S\left(\vec{s}_{1}+\vec{r}^{\prime}\right)-i S^{*}\left(\vec{s}_{1}\right)} \\
& \times R_{i n}\left(\vec{q}_{0}-\vec{q}_{1}, \vec{r}^{\prime}, 0\right) X_{f}\left(\overrightarrow{r^{\prime}}, \overrightarrow{r_{1}^{\prime}}, \overrightarrow{r_{1}^{\prime \prime}}\right) d^{2} r^{\prime} d^{2} s_{1} d^{2} s_{0},
\end{aligned}
$$

where

$$
\begin{aligned}
X_{f}\left(\vec{r}^{\prime}, \vec{r}_{1}^{\prime}, \vec{r}_{1}^{\prime \prime}\right)= & \left.\frac{T_{A} T_{P} P_{A} P_{P}}{4} \sqrt{1+P_{m}\left(\vec{s}_{1}+\vec{r}^{\prime}\right.}\right) \sqrt{1+P_{m}\left(\vec{s}_{1}\right)} \\
& \times \int_{A_{d}} \cos \left(\phi_{\mathrm{I}}+\phi_{\mathrm{II}}+\gamma_{m}\left(\vec{s}_{1}+\vec{r}^{\prime}\right)\right. \\
& \left.+\gamma_{m}\left(\vec{s}_{1}\right)\right) e^{-i \vec{q}_{d} \cdot \vec{r}^{\prime}} d^{2} r_{d},
\end{aligned}
$$

which, after filling in $\phi_{\mathrm{I}}+\phi_{\mathrm{II}}=\vec{l}_{s e} \cdot \vec{q}_{s e}, \vec{q}_{s e}=\vec{q}_{d}+\vec{q}_{0}-\vec{q}_{1}\left(r_{s} / r_{d}\right.$
$+1)$ and if the detector area is large enough, can be replaced by

$$
\begin{aligned}
X_{f}\left(\overrightarrow{r^{\prime}}, \overrightarrow{r_{1}^{\prime}}, \overrightarrow{r_{1}^{\prime \prime}}\right)= & \frac{T_{A} T_{P} P_{A} P_{P}}{4} \frac{4 \pi^{2} r_{d}^{2}}{\bar{k}^{2}} \sqrt{1+P_{m}\left(\vec{s}_{1}+\vec{r}^{\prime}\right)} \sqrt{1+P_{m}\left(\vec{s}_{1}\right)} \\
& \times \Re\left\{e^{i l_{s e} \cdot\left[\vec{q}_{0}-\vec{q}_{1}\left(r_{s} / r_{d}+1\right)\right]+i \gamma_{m}\left(\vec{s}_{1}+\vec{r}^{\prime}\right)+i \gamma_{m}\left(\vec{s}_{1}\right)}\right. \\
& \left.\times \delta^{(2)}\left(\vec{r}^{\prime}-\vec{l}_{s e}\right)\right\},
\end{aligned}
$$

giving for the flip count rate for a completely incoherent source:

$$
\frac{I_{f}}{I_{0}}=\Re\left\{\int_{A_{s}} e^{2 i \gamma_{m}\left(\vec{s}_{1}\right)} H_{r}\left(\vec{s}_{1}+\vec{l}_{s e}\right) H_{r}\left(\vec{s}_{1}\right) * d^{2} s_{1}\right\},
$$

where

$$
H_{r}(\vec{r})=\sqrt{\frac{1+P_{m}(\vec{r})}{2}} e^{i S(\vec{r})+i \gamma_{m}(\vec{r})} .
$$

The measured polarization is determined by the z-rotation part, $\hat{T}_{z}$, of the scattering matrix. The flip part $\hat{F}_{z}$ is completely depolarized. If a flipper [represented by a flipping matrix $\left.\hat{F}_{z}(2 \tau)\right]$ is inserted between region I and the sample, the scattering matrix is replaced by $\hat{H}_{f}=\hat{H}_{m} \hat{F}_{z}(2 \tau)$ which can be accounted for by replacement of the following variables:

$$
\begin{gathered}
P_{f, p}(\vec{r})=-P_{m}(\vec{r}), \\
\gamma_{f, p}(\vec{r})=\delta_{m}(\vec{r})-\tau-\pi, \\
\delta_{f, p}(\vec{r})=\gamma_{m}(\vec{r})+\tau .
\end{gathered}
$$

This means that by flipping the neutron spin just before the sample, the correlation function that is measured changes from the $\hat{T}_{z}$ part of the scattering matrix to the $\hat{F}_{z}$ part. The same holds for a flipper between the sample and region II:

$$
\begin{gathered}
P_{f, a}(\vec{r})=-P_{m}(\vec{r}), \\
\gamma_{f, a}(\vec{r})=-\delta_{m}(\vec{r})+\tau+\pi, \\
\delta_{f, a}(\vec{r})=-\gamma_{m}(\vec{r})+\tau .
\end{gathered}
$$

\section{Magnetic scattering in domains}

For a sample consisting of domains of some size and shape, the scattering matrix must be calculated using the procedure shown in Fig. 1, where now each region represents a single domain. Using Eq. (26) and the definition of the angles $\theta_{i}$ and $\vartheta_{i}$ according to Eq. (15):

$$
\hat{H}_{m}(\vec{r})=\prod_{j=1}^{N} \hat{T}_{z}\left(\vartheta_{j}\right)^{\dagger} \hat{T}_{y}\left(\theta_{j}\right)^{\dagger} \hat{T}_{z}\left(\phi_{j}\right) \hat{T}_{y}\left(\theta_{j}\right) \hat{T}_{z}\left(\vartheta_{j}\right)
$$

where $\quad \phi_{j}=-c B^{\prime}\left(\vec{r}_{j}+x_{j} \vec{e}_{x}\right) \bar{\lambda}\left(x_{j+1}-x_{j}\right), \quad c=-4 \pi m g_{r} \mu_{N} / h^{2}$ $=4.63209 \times 10^{14} \mathrm{~T}^{-1} \mathrm{~m}^{-2}$, and $B^{\prime}\left(\vec{r}_{j}\right)$ is the pseudomagnetic flux density ${ }^{5}$ in domain $j$ : 


$$
B^{\prime}(\vec{r})=-\frac{\rho_{m}(\vec{r}) h^{2}}{2 \pi g_{r} \mu_{N} m}=\frac{2 \rho_{m}(\vec{r})}{c},
$$

where $\rho_{m}(\vec{r})$ is the magnetic scattering length density so that

$$
\phi_{j}=-2 \bar{\lambda} \rho_{m}\left(\vec{r}_{j}+x_{j} \vec{e}_{x}\right)\left(x_{j+1}-x_{j}\right) .
$$

Using the fact that $\left(n_{x} \hat{\sigma}_{x}+n_{y} \hat{\sigma}_{y}+n_{z} \hat{\sigma}_{z}\right)^{2}=\hat{I}$, Eq. (137) can be reduced to

$$
\hat{H}_{m}(\vec{r})=\prod_{j=1}^{N} e^{i(c \bar{\lambda} / 2)\left(x_{j+1}-x_{j}\right)\left[B_{x}^{\prime}\left(x_{j}\right) \hat{\sigma}_{x}+B_{y}^{\prime}\left(x_{j}\right) \hat{\sigma}_{y}+B_{z}^{\prime}\left(x_{j}\right) \hat{\sigma}_{z}\right]},
$$

where the dependence of $B^{\prime}$ on $\vec{r}_{j}+x_{j} \vec{e}_{x}$ is written as $B^{\prime}\left(x_{j}\right)$. This equation can be approximated when $\phi_{j} \ll 1$ or $\vec{n}_{j} \approx \vec{n}_{j+1}$ by

$$
\hat{H}_{m}(\vec{r})=e^{i\left[\zeta_{x}(\vec{r}) \hat{\sigma}_{x}+\zeta_{y}(\vec{r}) \hat{\sigma}_{y}+\zeta_{z}(\vec{r}) \hat{\sigma}_{z}\right]},
$$

where $\zeta_{k}$ is a measure for the average pseudomagnetic flux density in the $k$ direction along the neutron beam:

$$
\zeta_{k}(\vec{r})=\frac{c \bar{\lambda}}{2} \sum_{j=1}^{N} B_{k}^{\prime}\left(\vec{r}+x_{j} \vec{e}_{x}\right)\left(x_{j+1}-x_{j}\right)=\frac{c \bar{\lambda} d_{x}}{2}\left\langle B_{k}^{\prime}(\vec{r})\right\rangle,
$$

where $k$ represents the indices $x, y$, or $z$. Equation (141) can be expanded to

$$
\hat{H}_{m}(\vec{r})=\hat{I} \cos \zeta(\vec{r})+i\left(\zeta_{x}(\vec{r}) \hat{\sigma}_{x}+\zeta_{y}(\vec{r}) \hat{\sigma}_{y}+\zeta_{z}(\vec{r}) \hat{\sigma}_{z}\right) \frac{\sin \zeta(\vec{r})}{\zeta(\vec{r})},
$$

where $\zeta(\vec{r})=\sqrt{\zeta_{x}(\vec{r})^{2}+\zeta_{y}(\vec{r})^{2}+\zeta_{z}(\vec{r})^{2}}$. Note that any rotation matrix can be put in this form. The exact interpretation of $\zeta_{x}$, $\zeta_{y}$, and $\zeta_{z}$ depends on the properties of the sample and the validity of the above or other approximations. The relation between $\zeta_{x}, \zeta_{y}, \zeta_{z}$ and $P_{m}, \gamma_{m}, \delta_{m}$ using the notation of Eq. (124) is

$$
\begin{gathered}
e^{i \gamma_{m}}=\frac{\zeta \cos \zeta+i \zeta_{z} \sin \zeta}{\sqrt{\zeta^{2} \cos ^{2} \zeta+\zeta_{z}^{2} \sin ^{2} \zeta}}, \\
e^{i \delta_{m}}=\frac{\zeta_{x}-i \zeta_{y}}{\sqrt{\zeta_{x}^{2}+\zeta_{y}^{2}}}, \\
P_{m}=1-2\left(\zeta_{y}^{2}+\zeta_{x}^{2}\right) \frac{\sin ^{2} \zeta}{\zeta^{2}} .
\end{gathered}
$$

Now, Eq. (129) becomes

$$
\frac{I_{f}}{I_{0}}=\Re\left\{\int_{A_{s}} P_{r}\left(\vec{s}_{1}\right) H_{r}\left(\vec{s}_{1}+\vec{l}_{s e}\right) H_{r}\left(\vec{s}_{1}\right) * d^{2} s_{1}\right\},
$$

where

$$
P_{r}(\vec{r})=\frac{\left[\zeta(\vec{r}) \cos \zeta(\vec{r})+i \zeta_{z}(\vec{r}) \sin \zeta(\vec{r})\right]^{2}}{\zeta(\vec{r})^{2} \cos ^{2} \zeta(\vec{r})+\zeta_{z}(\vec{r})^{2} \sin ^{2} \zeta(\vec{r})},
$$

and

$$
H_{r}(\vec{r})=\left(\cos \zeta(\vec{r})+i \zeta_{z}(\vec{r}) \frac{\sin \zeta(\vec{r})}{\zeta(\vec{r})}\right) e^{i S(\vec{r})}
$$

Note that $P_{r}$ does not depend on the spin echo length. If a flipper is inserted $(\tau=n \pi)$ between region I and the sample, the flip count rate can be found by using Eqs. (131)-(133):

$$
\frac{I_{f, f}}{I_{0}}=\Re\left\{\int_{A_{s}} P_{r, f}\left(\vec{s}_{1}\right) H_{r, f}\left(\vec{s}_{1}+\vec{l}_{s e}\right) H_{r}\left(\vec{s}_{1}\right) * d^{2} s_{1}\right\},
$$

where

$$
P_{r, f}(\vec{r})=\frac{\left[\zeta_{x}(\vec{r})-i \zeta_{y}(\vec{r})\right]^{2}}{\zeta_{x}(\vec{r})^{2}+\zeta_{y}(\vec{r})^{2}}
$$

and

$$
H_{r, f}(\vec{r})=\left[\zeta_{x}(\vec{r})-i \zeta_{y}(\vec{r})\right] \frac{\sin \zeta\left(\vec{s}_{1}\right)}{\zeta\left(\vec{s}_{1}\right)} e^{i S(\vec{r})}
$$

For spin echo lengths where $H_{r}\left(\vec{s}_{1}+\vec{l}_{s e}\right) \approx H_{r}\left(\vec{s}_{1}\right)$ and the same for $H_{r, f}$, this equation can be reduced further. If also the nuclear scattering is neglected, the measured polarizations are given by

$$
\begin{aligned}
& \frac{P_{m}}{P_{A} P_{P}}=\left\langle\cos ^{2} \zeta\left(\vec{s}_{1}\right)-\zeta_{z}\left(\vec{s}_{1}\right)^{2} \frac{\sin ^{2} \zeta\left(\vec{s}_{1}\right)}{\zeta\left(\vec{s}_{1}\right)^{2}}\right\rangle_{A_{s}}, \\
& \frac{P_{m, f}}{P_{A} P_{P}}=\left\langle\left[\zeta_{x}\left(\vec{s}_{1}\right)^{2}-\zeta_{y}\left(\vec{s}_{1}\right)^{2}\right] \frac{\sin ^{2} \zeta\left(\vec{s}_{1}\right)}{\zeta\left(\vec{s}_{1}\right)^{2}}\right\rangle_{A_{s}} .
\end{aligned}
$$

One should realize, however, that as soon as the value of $\phi_{I}$ or $\phi_{\text {II }}$ reduces to almost 0 (hence for small spin-echo lengths), the above approximation (ignoring the terms which contain $\phi_{1}-\phi_{2}$ ) is not valid any more. An example of such a measurement is the so-called neutron depolarization technique. ${ }^{32}$ In this case, the flip count rate should be evaluated differently from the one represented before. The term containing $\phi_{2}-\phi_{1}$ in Eq. (125) should also be taken into account so that the four-flip count rate becomes

$$
\begin{aligned}
\frac{I_{f}}{I_{0}}= & \int_{A_{s}} e^{i S\left(\vec{s}_{1}\right)-i S^{*}\left(\vec{s}_{1}\right)}\left(\frac{1+P_{m}\left(\vec{s}_{1}\right)}{2} \cos \left(2 \gamma_{m}\left(\vec{s}_{1}\right)\right)\right. \\
& \left.-\frac{1-P_{m}\left(\vec{s}_{1}\right)}{2} \cos \left(2 \delta_{m}\left(\vec{s}_{1}\right)\right)\right) d^{2} s_{1} .
\end{aligned}
$$

From this equation, the four-measured polarization can be evaluated using the results of the previous section [Eqs. (144)-(146)] and neglecting the nuclear scattering:

$$
\frac{P_{m}}{P_{A} P_{P}}=\left\langle 1-2\left[\zeta_{x}\left(\vec{s}_{1}\right)^{2}+\zeta_{z}\left(\vec{s}_{1}\right)^{2}\right] \frac{\sin ^{2} \zeta\left(\vec{s}_{1}\right)}{\zeta\left(\vec{s}_{1}\right)^{2}}\right\rangle_{A_{s}},
$$

different from the one derived in the previous section. This corresponds to the $z z$ element of the depolarization matrix as found in Sec. V A. In this case, introducing an extra $\pi$ flipper between region I and the sample just reverses the sign of the measured polarization. 


\section{CONCLUSION}

It is possible to use coherence theory to describe propagation of neutrons through neutron scattering instruments. Coherence theory describes the propagation of the ensemble average of the neutron wave function. Coherence theory as adopted here only considers neutron wave functions, having approximately an equal amount of total energy, denoted by quasimonochromatic wave functions. This is due to the dispersion relation of matter waves, and hence the interference between nonmonochromatic wave functions can, in general, be ignored.

One advantage using coherence theory over standard scattering theoretical considerations is the incorporation of instrumental and source effects. By means of coherence theory, it is possible to accurately calculate scattered neutron intensities given the full instrumental details. It was shown that it is possible to retrieve the results as published in literature by introducing appropriate approximations. The introduced approximations are documented so one can assess when a particular approximation is valid and when it is not. Further, it was shown that also neutron polarization effects can be described by using the coherence matrix approach. This enables understanding of beam splitting effects due to magnetic flux density variations and indicates when these effects will become really measurable in real neutron scattering devices.

Another advantage of coherence theory is that, in principle, no conversion to momentum space (or $q$ space) is needed. All scattering phenomena can be described in real space. The Fourier transform to go from real space to momentum space and vice versa is contained in the propagation formula for the mutual coherence function or coherence matrix. This formula describes the propagation of the mutual coherence function through free space (vacuum or small interaction potential). It must be emphasized that scattering of neutrons is not incorporated in the coherence theory. It only deals with propagation of neutrons before interaction with a scattering object and after it.

Interaction with the object itself is described by means of the time-dependent Schrödinger equation for a (polarized) neutron wave function. Here, coherence lengths and time do not play any role because these are related to coherence properties only, not to the wave function. Hence, one should realize that a neutron wave function "scatters" from the whole of the interaction potential, not just from the part of the interaction potential covered by the coherence lengths (coherence volume) and time of the neutron ensemble average (or neutron beam). Although this seems obvious at first, it can become confusing when trying to understand the scattering of neutrons in real space.

Coherence theory seems to be quite complicated and unnecessary to describe the neutron propagation through an instrument. However, after getting used to the concepts of the mutual coherence function and the way this function is propagated through free space and space with magnetic flux density, it can give a more thorough account of the important effects before and after the scattering process. The phaseobject approximation (used for small angle neutron scattering) is extended to incorporate magnetic scattering. It is shown that the combination of the phase-object approximation and coherence matrix propagation is a powerful tool to determine the observables in SESANS instruments and the depolarization technique.

\section{ACKNOWLEDGMENTS}

This work was financially supported by the Nederlandse Organisatie voor Wetenschappelijk Onderzoek (NWO). This research project has been supported in part by the European Commission under the 6th Framework Program through the Key Action Strengthening the European Research Area, Research Infrastructures (Contract No. RII3-CT-2003-505925).
*Also at BonPhysics Research and Investigations B.V., Laan van Heemstede 38, 3297 AJ, Puttershoek, The Netherlands; victor@bonphysics.nl

${ }^{1}$ L. Mandel and E. Wolf, Optical Coherence and Quantum Optics, 1st ed. (Cambridge University Press, Cambridge, 1995).

${ }^{2}$ V. Holý, U. Pietsch, and T. Baumbach, High-Resolution X-ray Scattering from Thin Films and Multilayers (Springer-Verlag, Berlin, 1999).

${ }^{3}$ S. K. Sinha, M. Tolan, and A. Gibaud, Phys. Rev. B 57, 2740 (1998).

${ }^{4}$ F. van der Veen and F. Pfeiffer, J. Phys.: Condens. Matter 16, 5003 (2004).

${ }^{5}$ V. F. Sears, Neutron Optics, 1st ed. (Oxford University Press, New York, 1989).

${ }^{6}$ H. Rauch, H. Wölwitsch, H. Kaiser, R. Clothier, and S. A. Werner, Phys. Rev. A 53, 902 (1996).

${ }^{7}$ R. Gähler, J. Felber, F. Mezei, and R. Golub, Phys. Rev. A 58, 280 (1998).

${ }^{8}$ V. O. de Haan, J. Plomp, W. G. Bouwman, M. Trinker, M. T.
Rekveldt, C. P. Duif, E. Jericha, H. Rauch, and A. A. van Well, J. Appl. Crystallogr. 40, 151 (2007).

${ }^{9}$ F. Mezei, in Imaging Processes and Coherence in Physics, Lecture Notes in Physics Vol. 112, edited by M. Schlenker (Springer-Verlag, Berlin, 1980), p. 282.

${ }^{10}$ P. A. M. Dirac, Proc. R. Soc. London 117, 610 (1928).

${ }^{11}$ F. Mezei, Physica B \& C 151, 74 (1988).

${ }^{12}$ W. H. Kraan, Ph.D. thesis, Delft University of Technology, 2004.

${ }^{13}$ G. Arfken, Special Unitary Group, and Homomorphism. Mathematical Methods for Physicists, 3rd ed. (Academic, Orlando, FL, 1985).

${ }^{14}$ C. Schwink and O. Schärpf, Z. Phys. B 21, 305 (1975).

${ }^{15}$ R. P. Feynman, Rev. Mod. Phys. 20, 367 (1948).

${ }^{16}$ L. I. Schiff, Quantum Mechanics (McGraw-Hill, New York, 1968).

${ }^{17}$ B. Jap and R. Glaeser, Acta Crystallogr., Sect. A: Cryst. Phys., Diffr., Theor. Gen. Crystallogr. 34, 94 (1978).

${ }^{18}$ M. T. Rekveldt, Nucl. Instrum. Methods Phys. Res. B 114, 366 (1996). 
${ }^{19}$ M. T. Rekveldt, J. Appl. Crystallogr. 36, 1301 (2003).

${ }^{20}$ M. T. Rekveldt, W. G. Bouwman, W. H. Kraan, O. Uca, S. Grigoriev, S. Habicht, and T. Keller, in Neutron Spin Echo, Lecture Notes in Physics Vol. 601, edited by F. Mezei, C. Pappas, and T. Gutberlet (Springer, Berlin, 2003), Chap. 8, pp. 87-99.

${ }^{21}$ R. Gähler, R. Golub, K. Habicht, T. Keller, and J. Felber, Physica B 229, 1 (1996).

${ }^{22}$ E. Jericha, M. Baron, M. Hainbuchner, R. Loidl, M. Villa, and H. Rauch, J. Appl. Crystallogr. 36, 778 (2003).

${ }^{23}$ M. Agamalian, G. D. Wignall, and R. Triolo, J. Appl. Crystallogr. 30, 345 (1997).

${ }^{24}$ J. G. Barker, C. J. Glinka, J. J. Moyer, M. H. Kim, A. R. Drews, and M. Agamalian, J. Appl. Crystallogr. 38, 1004 (2005).

${ }^{25}$ R. Gähler, R. Golub, K. Habicht, T. Keller, J. Felber, and F.
Mezei, Physica B 229, 1 (1996).

${ }^{26}$ K. Habicht, R. Golub, R. Gähler, and T. Keller, in Neutron Spin Echo Spectroscopy: Basics, Trends and Applications, Lecture Notes in Physics Vol. 601 edited by F. Mezei, C. Pappas, and T. Gutberlet (Springer, Berlin, 2002), pp. 116-132.

${ }^{27}$ F. Mezei, Z. Phys. 255, 146 (1972).

${ }^{28}$ R. Pynn, J. Phys. E: J. Sci. Instrum. 11, 1133 (1978).

${ }^{29}$ T. Keller, R. Gähler, H. Kuntze, and R. Golub, Neutron News 6, 16 (1995).

${ }^{30}$ S. V. Grigoriev, W. H. Kraan, M. T. Rekveldt, T. Kruglov, and W. G. Bouwman, J. Appl. Crystallogr. 39, 252 (2006).

${ }^{31}$ M. T. Rekveldt, S. V. Grigoriev, W. H. Kraan, N. H. van Dijk, and W. G. Bouwman, Rev. Sci. Instrum. 77, 073902 (2006).

${ }^{32}$ M. T. Rekveldt, Z. Phys. 259, 391 (1973). 